\title{
Le terme et le début du Naufragé
}

Par

\section{Claude Obsomer*}

Université de Namur, Université catholique de Louvain

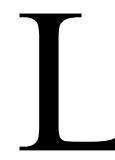

e récit du Naufragé est connu par une copie unique, celle du papyrus $n^{\circ} 1115 \mathrm{du}$ musée de l'Ermitage à Saint-Pétersbourg ${ }^{1}$. On ignore la provenance de ce papyrus et les circonstances de son entrée au musée, où il fut découvert début 1881 et déroulé sans trop de difficultés. C'est en septembre 1881, lors du Cinquième Congrès international des Orientalistes qui se tenait à Berlin, que Wladimir Golénischeff révéla l'existence de ce texte littéraire du Moyen Empire qui lui semblait offrir des similitudes avec les récits des voyages d'Ulysse et de Sindbad le Marin ${ }^{2}$. Golénischeff publia une première traduction du Naufragé,

* Je remercie Bernard Mathieu pour les données bibliographiques communiquées, ainsi que Jean-Claude Haelewyck et Daniel Malnati pour leur relecture attentive.

${ }^{1}$ Coupé en huit sections, le papyrus a une longueur de $380 \mathrm{~cm}$ et une hauteur de $12 \mathrm{~cm}$ environ, d'après GOLÉNISCHEFF 1913, p. 2.

${ }^{2}$ GOLÉNISCHEFF 1882a. Il pensait plus précisément au séjour d'Ulysse dans l'île d'Alcinoos (p. 107-110) et à celui de Sindbad dans l'île du roi Mihradj (p. 113-117), tous deux consécutifs à un naufrage. 
en français, dès $1882^{3}$, mais il attendit plus de vingt ans pour éditer le texte en transcription hiéroglyphique ${ }^{4}$. Cette editio princeps, qui respecte la disposition du texte en colonnes et en lignes ${ }^{5}$, devança de peu l'édition d'Adolf Erman, effectuée sur base de photographies, qui dispose le texte en fonction de sa structure grammaticale ${ }^{6}$. C'est une édition semblable à celle d'Erman que Golénischeff proposa en 1912 dans son livre intitulé Le Conte du Naufragé, qui consiste pour l'essentiel en un glossaire dont les termes donnent lieu à de larges commentaires ${ }^{7}$. L'année suivante parut la reproduction photographique du papyrus, qui offrait désormais un accès direct à l'original en écriture hiératique ${ }^{8}$. Ces premières éditions furent suivies par celles d'Aylward M. Blackman en 1932 et d'Adriaan De Buck dès 1941 ${ }^{9}$, ouvrages qui assurèrent le succès du Naufragé dans l'enseignement académique de la langue égyptienne. En 1958, W. Kelly Simpson a identifié une allusion au Naufragé dans un texte ramesside ${ }^{10}$.

Rédigé dans la langue classique qu'est le moyen égyptien, avec peu de fautes manifestes dans l'unique copie conservée, le Naufragé est l'un des textes les plus étudiés du Moyen Empire. Il offre une structure narrative remarquable, constituée de trois récits enchâssés ${ }^{11}$. Le premier consiste en des propos tenus par un $\check{s} m s w i k r$ anonyme à son supérieur, un $h^{3}{ }^{\dagger} y-{ }^{-}$tout aussi anonyme ${ }^{12}$, au retour d'une expédition navale en Ouaouat (Nubie), une fois leur bateau

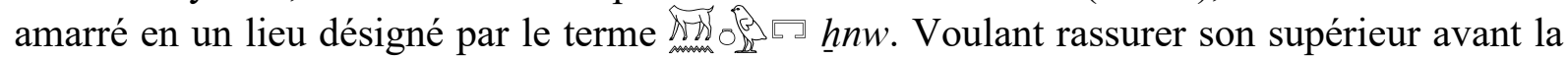
réception de celui-ci par le roi, lui aussi anonyme, le šmsw en vient à lui raconter une mésaventure qui lui est advenue jadis tandis qu'il naviguait sur Ouadj-our pour gagner la « région minière $(b i 3)$ », à identifier au Sinaï comme nous le confirmerons plus loin. Tel est l'objet de ce deuxième récit ${ }^{13}$ : après un naufrage, dont il était sorti seul survivant, il avait abouti sur une île merveilleuse dont le maître, un gigantesque serpent, avait fini par l'accueillir et le rassurer sur son avenir. Le troisième récit ${ }^{14}$, assez bref, est celui du Serpent, qui évoquait

\footnotetext{
${ }^{3}$ GolÉNISCHEFF 1882a, p. 101-106 ; 1882b, p. 341-344. Traduction revue : GOLÉNISCHEFF 1891, p. 177-182. On notera aussi la traduction en russe publiée par STASOW 1882, p. 582-586.

${ }^{4}$ GOLÉNISCHEFF 1906, p. 75-84, avec traduction. L'auteur ajoute des annotations nombreuses incluant des extraits de l'Odyssée et des Contes des Mille et Une Nuits.

${ }^{5}$ Le texte se compose de 123 colonnes verticales (1-123), suivies de 53 lignes horizontales (124-176), pour se terminer par 13 colonnes verticales (177-189).

${ }^{6}$ ERMAN 1906, p. 4-25, avec une traduction commentée. SETHE 1907, p. 80-87, compléta cette publication d'une série de remarques philologiques.

${ }^{7}$ GOLÉNISCHEFF 1912.

${ }^{8}$ GOLÉNISCHEFF 1913, pl. I-VIII. Une transcription en hiéroglyphes figure en vis-à-vis de chaque planche.

${ }^{9}$ BLACKMAN 1932, p. 41-48; DE BUCK 1941, p. 8-20 (avec translittération); DE BUCK 1948, p. $100-106$ (3 édition en 1970).

${ }^{10}$ Ostracon OIC 12074, ligne 4 (allusion à Naufragé 30-31, 97-98) : SIMPSON 1958, p. 50 ; FosTER 1984, p. 90-93, GUGLIELMI 1984, p. 352-353 ; VERNUS 2013, p. 200-201. Pour une référence possible à Naufragé 6970 et 83-86 dans l'inscription de la stèle de Piânkhy, voir GugLIELMI 1984, p. 360-362. Voir aussi MoERS 2001, p. 131-137; DORN 2013, p. 96.

${ }^{11}$ Voir aussi KURTH 1987, p. 176 ; BAINES 1990, p. 67 ; CANNUYER 1998, p. 30 ; SIMPSON 2003, p. 46.

${ }^{12}$ Le caractère anonyme de ces personnages convient bien à un récit de fiction : voir BAINES 1990, p. 58. Leurs titres sont attestés dans certaines inscriptions d'expéditions du Moyen Empire: voir ENMARCH 2001, p. 100-102.

13 À partir de la colonne 21 : « Je vais donc te raconter une chose semblable qui m'est arrivée à moi-même ».

14 À partir de la ligne 125 : « Je vais donc te raconter une chose semblable qui s'est produite dans cette île ».
} 
devant le Naufragé le cataclysme qui avait emporté sa famille, le laissant seul sur l'Île. La structure d'ensemble peut être résumée visuellement comme suit :

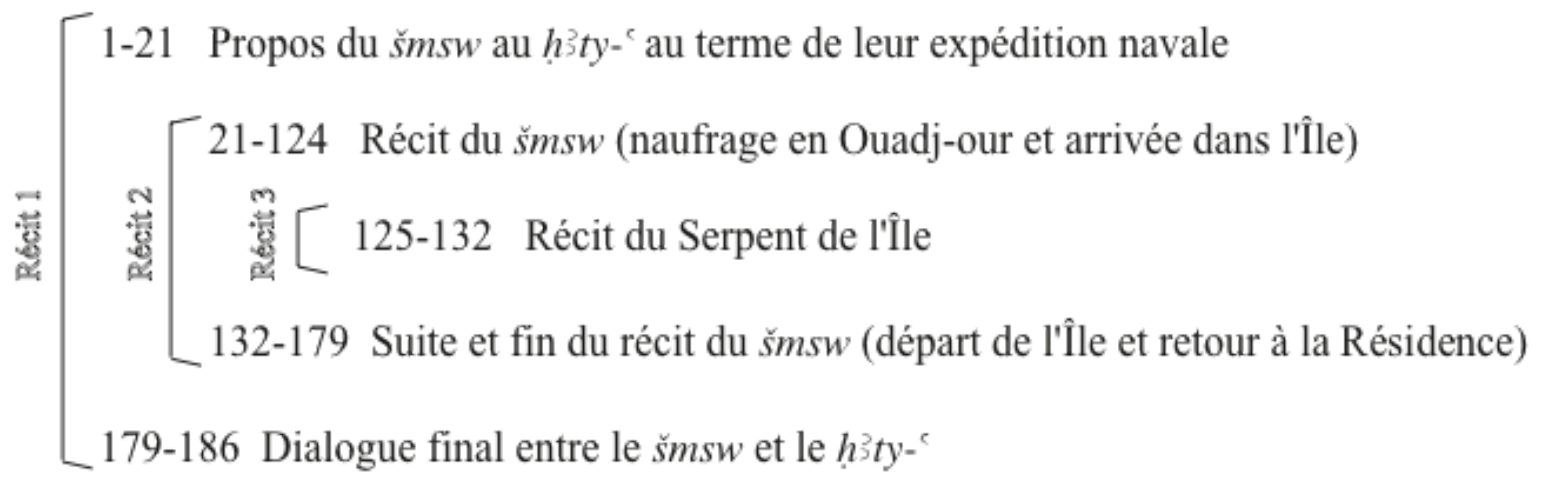

L'objet de cet article sera de réexaminer les onze premières colonnes du texte, qui évoquent l'expédition navale au terme de laquelle le $\check{s} m s w$ entreprend de raconter sa mésaventure en Ouadj-our. Le but sera d'établir le sens précis du terme $h n w$ de la phrase (col. 2-3) en vue de déterminer l'endroit du cours du Nil où le bateau est censé être amarré, avant la réception du ḩ̉ty- ' par le roi. En effet, comme c'est le cas pour le récit de la fuite de Sinouhé, l'auteur semble avoir été animé par le souci de poser un cadre spatio-temporel réaliste et précis ${ }^{15}$, même si les faits et personnages sont fictifs. Avant de relire le passage et les traductions proposées, il n'est pas inutile de rappeler brièvement, en vue d'éclairer le propos, ce que nous savons aujourd'hui des expéditions navales égyptiennes du Moyen Empire, à destination de la Nubie, du Sinaï et de Pount ${ }^{16}$.

\section{Données sur les expéditions navales au Moyen Empire}

\section{a. Le récit 2}

En énonçant le contexte de son naufrage passé, le šmsw dit au $h^{3}{ }^{\top}{ }^{-}{ }^{c}$ : « J'étais parti vers la région minière du souverain / pour le souverain ${ }^{17}$. J'étais descendu sur Ouadj-our dans un bateau de 120 coudées de long, etc. » (col. 23-26: Šm.kwi $r$ bỉ $n(y) / n$ ity. H3.kwi $r W^{3} \underline{d}$-wr $m$ dpt $n(y) t m h 120 \mathrm{~m} 3$ sw.s, ...). Plus loin, il est plus concis en s'adressant au Serpent : «J'étais descendu vers la région minière en mission du souverain dans un bateau de 120 coudées de long, etc. » (col. 89-91 : H3.kwi $r$ bỉ $m$ wpwt ity $m$ dpt $n(y) t ~ m h 120$ m 3w.s, ...). Les termes $b i 3$ et $W 3 \underline{d}-w r$ sont à expliquer.

Erman fut le premier à proposer de situer $b i 3$ au Sinaï ${ }^{18}$, alors que Golénischeff pensait à une localisation «dans le voisinage immédiat du pays de Pount ${ }^{19}$. Le terme bỉ a été relevé

\footnotetext{
15 Voir Posener 1938, p. 266-267.

${ }^{16}$ En ce qui concerne la Nubie et Pount, je renvoie à mes publications précédentes pour éviter d'allonger cette section.

${ }^{17}$ Construction génitivale dans la plupart des traductions publiées. Mais chez GARDINER, PEET, ČERNÝ 1955, p. 2, le signe $n$ est compris comme un datif « pour le souverain », l'expression ayant la même signification que $m$ wpwt ity de la col. 90. Choix identique dans SIMPSON 1972, p. 51 ; 2003, p. 47 ; GoEDICKE 1974, p. 14 ; Bradbury 1988, p. 139, n. 59 ; GAlÁN 1998, p. 35 ; 2005, p. 31 ; CASEY 2008, p. 32 ; Allen 2015, p. 15-16.

${ }^{18}$ ERMAN 1906, p. 7 : «Das "Bergwerk des Königs" wird eine Sinaigrube sein ». ERMAN 1923, p. 58, n. 2 : «Er fährt von einem Hafen des Roten Meeres zu den Gruben der Sinaihalbinsel ». Cette idée fut adoptée par
} 
dans cinq inscriptions du Sérabit el-Khadim datées ou datables du règne d'Amenemhat III. Quatre d'entre elles mentionnent la venue de quelqu'un vers $b i 3$ $p n$ « ce bia » ou « cette région minière $»^{20}$ : on peut en déduire que le Sérabit el-Khadim est une région minière parmi d'autres $^{21}$. La cinquième (IS 141) peut être attribuée à l'expédition de l'an 7 d'Amenemhat III grâce à la mention du gardien du cellier $\left({ }^{i} r y-{ }^{\circ} t\right)$ Ânkhreni, mentionné aussi sur la petite stèle IS $401^{22}$. Le chef d'expédition, le chancelier du dieu Amenemhat, déclare ${ }^{23}:$ «Je suis venu à la région minière $\left(r b b^{3}\right)$ pour mon maître $(n n b . i) /$ de mon maître $(n(y) n b . i)$ ). La première lecture est préconisée par les éditeurs ${ }^{24}$, ce qui permet d'envisager $b i 3$ comme un toponyme à part entière, comme c'est le cas en Naufragé 90. Il en va logiquement de même pour l'inscription de l'an 9 de Sésostris $I^{\text {er }}$ découverte à Ayn Soukhna ${ }^{25}$, qui mentionne l'envoi de quelqu'un $r$ bi $n n s w$-bity $H p r-k \jmath-R^{c}$ «à la région minière pour le roi de Haute et de Basse Égypte Khéperkarê ${ }^{26}$. Dans son excellent ouvrage paru en 2018, Pierre Tallet identifie une quarantaine d'expéditions datées qui furent menées au Sinaï durant le Moyen Empire, les premières au départ d'Ayn Soukhna (an 1 de Mentouhotep IV, an 7 d'Amenemhat $I^{\text {er }}$, an 9 de Sésostris $\left.\mathrm{I}^{\mathrm{er}}\right)^{27}$. Il admet que les expéditions du règne de Sésostris $\mathrm{I}^{\mathrm{er}}$ ont pu être plus nombreuses, vu le nombre d'inscriptions de ce règne sans mention de l'année précise qui furent trouvées au Sérabit el-Khadim ${ }^{28}$. Par conséquent, le terme $b i 3$ mentionné à deux reprises dans le Naufragé semble bien désigner la zone de Sérabit el-Khadim dans le Sinaï, comme la région minière "par excellence » où se rendaient de très nombreuses expéditions du Moyen Empire $^{29}$. On retiendra qu'il est préférable de comprendre en Naufragé 23-24 «J'étais parti

WIEDEMANN 1906, p. 26 ; BUdGe 1914, p. 208, n. 1 ; RoEDER 1927, p. 18, et par la majorité des égyptologues qui suivirent.

${ }^{19}$ GOLÉNISCHEFF 1912, p. 60-62, qui se référait au passage de la lettre de Pépy II à Herkhouf où le roi dit à celui-ci : « Ma Majesté souhaite voir ce nain plus que les inw bỉ Pwnt» (Urk. I, 130.14-15). Mais il est préférable de voir ici deux termes coordonnés et de traduire « les produits de Bia et de Pount », comme préconisé par Breasted 1906, p. 161 (§ 353). Choix identique dans Posener 1938, p. 267 ; GARdiner, PeET, ČERnÝ 1955, p. 2 ; LiCHTHEIM 1973, p. 27, n. 7 ; TATERKA 2018, p. 49-50, 410 ; OBSOMER 2019a, p. 49 ; 2019b, p. 29. Sur le sens de bỉ Pwnt / bỉ n(y) Pwnt des documents de Mersa Gaouasis, voir TATERKA 2018, p. 410-412 ; OBSOMER 2019a, p. 49-50 ; 2019b, p. 29-30 ; TATERKA à paraître (je le remercie de m'avoir transmis son manuscrit pour lecture).

${ }^{20}$ Inscriptions IS 53 (1. 3), IS 90 (face ouest, 1. 2), IS 117 (face est, 1. 9-10) et IS 409 (1. 4). Voir GARDINER, PEET, ČERNÝ 1952, pl. XVII, XXVI, XL, LXXXIII.

${ }^{21}$ GARDINER, PEET, ČERNÝ 1955, p. 2.

22 SEYFRIED 1981, p. 183 ; TALLET 2018, p. 164-165.

${ }^{23}$ Inscription IS 141 (face ouest, 1. 5). Voir GARDINER, PEET, ČERNÝ 1952, pl. LII.

${ }^{24}$ Gardiner, PeEt, Černý 1955, p. 2, 140. Voir aussi Goedicke 1974, p. 17 ; TAllet 2018, p. 165.

${ }^{25}$ Inscription CCIS 220 : ABD EL-RAZIQ 2002, p. 57-58, fig. 28, ph. 71 (n²2); TALLET 2012, I, p. $202-203$; II, p. $142 ; 2018$, p. 313.

${ }^{26}$ Voir ABD EL-RAZIQ 2002, p. 57, où le $n$ est considéré comme la préposition du datif. Cependant, TALLET 2012, p. 202 ; 2018, p. 144, préfère y voir un génitif, comme il est de coutume pour Naufragé 23-24 : « le pays minier du roi de Haute et de Basse Égypte Kheperkarê ».

27 TALLET 2018, p. 139-206, 311-363.

28 TALLET 2018, p. 144.

${ }^{29}$ On ne suivra pas BRADBURY 1988, p. 139, n. 59, lorsqu'elle localise à Pount la région minière $b i 3$ mentionnée dans le Naufragé, sur base de la mention des deux mois de navigation, excessive pour se rendre au Sinaï. En effet, ces deux mois concernent le trajet entre l'Île du Serpent et la Résidence de Licht (Naufragé 167-168, 173-174) et non le trajet pour se rendre à la région minière $b i$ ? 
vers la région minière (bi3) pour le souverain ( $n$ ity) », en IS 141 «Je suis venu à la région minière $(b i j)$ pour mon maître $(n n b . \hat{l})$ », et en CCIS 220 « envoyer $\mathrm{N}$ à la région minière $(b i 3)$ pour le roi de Haute et de Basse Égypte Khéperkarê (n $n s w$-bity $H p r-k \jmath-R$ ) », en considérant qu'il s'agit chaque fois de la préposition $n$ du datif et non d'une construction génitivale avec $n(y)$.

Pour se rendre au Sinaï (bỉ), le héros descend sur Ouadj-our (col. 24-25 : H3.kwi $r$ W’ $\underline{d}$-wr $m d p t$ ) et embarque sur un bateau dont l'équipage est expérimenté. La tempête se déclenche alors que le bateau se trouve sur Ouadj-our (col. 33, 102 : iw. $n m W$ s $\underline{d}$-wr). Mais « alors qu'on supportait le vent, elle redoubla d'intensité, provoquant une vague- $n w y t$ de huit coudées $\gg{ }^{30}$, qui frappa le bateau de plein fouet et le fit sombrer. C'est une vague- $w \supset w$ de Ouadj-our qui amène alors le Naufragé vers l'Île (col. 39-41, 109-110), désignée plus tard par le Serpent comme « cette île de Ouadj-our dont les côtés sont dans le flot » (col. 84-86 : íw pn $n(y) W 3 \underline{d}$ $w r, n t y$ gs (wy).fy $m n w y)^{31}$.

Si les travaux de Claude Vandersleyen ont mis en évidence que le terme $W 3 \underline{\jmath} \underline{d}-w r$ ne signifie pas « la mer » dans un bon nombre d'occurrences, il est clair, comme j'ai pu le démontrer dès 1995, que l'inscription d'Amény découverte à Mersa Gaouasis (règne de Sésostris $\mathrm{I}^{\mathrm{er}}$ ) l'emploie pour désigner la mer Rouge ${ }^{32}$. Il en va de même dans l'inscription d'Hénou au Ouadi Hammamat (règne de Mentouhotep III) ${ }^{33}$, et dans l'inscription IS 211 du Sérabit el-Khadim (règne d'Aménophis III) $)^{34}$.

Les dimensions du bateau (120 coudées de long et 40 de large) sont fort exagérées, comme Maspero le notait déjà ${ }^{35}$. En effet, 120 coudées correspondent à un peu plus de 60 mètres, alors que Patrice Pomey a calculé que les pièces de bois de la XII ${ }^{\mathrm{e}}$ dynastie découvertes dans les galeries d'Ayn Soukhna avaient appartenu à des bateaux d'une longueur voisinant les 15 mètres ${ }^{36}$.

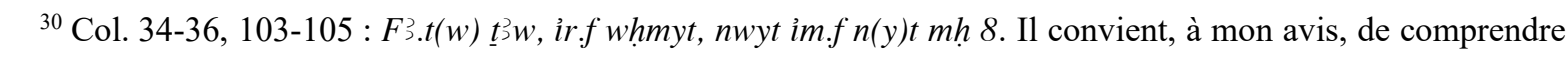
que ces marins expérimentés étaient à même d'utiliser un fort vent pour accélérer la traversée, mais qu'ils furent dépassés par les événements lorsque la tempête devint plus violente, provoquant la perte du navire. On ne verra donc aucune contradiction entre le fait qu'ils étaient d'excellents marins et le fait que le navire finit par sombrer.

${ }^{31}$ L'égyptien me semble employer le duel gswy comme il le fait pour $r d w y$ « les pieds », 'wy « les bras », etc. Aucune nécessité d'y voir une île de forme oblongue : le Serpent précise simplement que l'île est totalement entourée d'eau (cfr WESTENDORF 1990, p. 1060).

${ }^{32}$ Au départ de l'analyse des démonstratifs employés : OBSOMER 1995, p. 398-399; 2019a, p. 51 ; 2019 b, p. 28.

${ }^{33}$ Voir OBSOMER 2019b, p. 13-16, 28-29, 34-36.

${ }^{34}$ C'est aussi l'avis de CANNUYER 2019, p. 27, pourtant ardent défenseur de l'interprétation nilotique de Vandersleyen. L'inscription IS 211 (GARDINER, PEET, ČERNÝ 1955, pl. LXVI) fera l'objet d'une publication dans un prochain numéro du $B A B E L A O$.

${ }^{35}$ MASPERO 1905 , p. 86 , n. 2 ; 1911, p. 107, n. 3 : « Le navire de notre conte appartient (...) à la classe des vaisseaux invraisemblables dont on trouve tant d'exemples dans les traditions populaires de tous les pays ». Pour une lecture symbolique du nombre 120 et des autres nombres mentionnés dans le Naufragé, voir CANNUYER 1998, p. 31-33 ; DESROCHES-NOBLECOURT 1998, p. 63-64.

${ }^{36}$ POMEY 2011, p. 10. On notera que les dimensions indiquées dans le Naufragé (120 coudées de long et 40 coudées de large) sont identiques à celles qui seront renseignées dans la biographie d'Inéni pour le navire destiné au transport d'obélisques sous Touthmosis $\mathrm{I}^{\mathrm{er}}$ : Urk. IV, 56.13-15. De grands bateaux sont attestés dès l'Ancien Empire : un bateau de 100 coudées est mentionné dans la Pierre de Palerme pour le règne de Snéfrou (Urk. I, 
Quand le Serpent annonce au Naufragé qu'il rentrera chez lui après quatre mois passés sur l'Île, celui-ci promet de lui faire parvenir des produits d'Égypte parmi lesquels de l'huilehékénou et de l'encens-sntr. Mais le Serpent lui répond de façon ironique : "C'est moi le souverain de Pount. La myrrhe-'ntyw m'appartient. Cette huile-hékénou dont tu as dit qu'elle serait apportée, c'est la production principale de cette île » (1.151-152 : 'Ink is hlk’ Pwnt. 'ntyw, $n . l i m(y)$ sw. $H k n w$ pf $\underline{d} d(w) . n . k$ in.t( $w) . f, b w$ pw wr $n(y)$ iw pn). C'est la seule mention de Pount dans le Naufragé.

Le Moyen Empire est la période qui connaît le plus grand nombre d'expéditions attestées vers Pount : celles-ci apparaissent comme des activités de prestige pour les rois qui les ont envoyées, car les produits de Pount étaient plus communément acheminés en Égypte par les voies du commerce africain. La plus ancienne eut lieu sous Mentouhotep III, mais on ignore si elle fut couronnée de succès car seules les opérations préalables à l'envoi des navires sont mentionnées dans l'inscription d'Hénou ${ }^{37}$. La seconde à être attestée (an 23/24 de Sésostris I ${ }^{\text {er }}$ ) est la mieux documentée. Elle est connue par les inscriptions de monuments commératifs découverts à Mersa Gaouasis, nouveau port intermittent de la mer Rouge sans doute inauguré à cette occasion : l'inscription d'Amény concerne les travaux préalables à l'envoi des bateaux ; les inscriptions d'Ânkhou détaillent sa mission vers Pount et les produits qui en furent rapportés, faisant état également de la présence royale à Mersa Gaouasis au retour de l'expédition ${ }^{38}$. Après Sésostris $\mathrm{I}^{\text {er }}$, chaque souverain enverra au moins une expédition vers Pount jusqu'au règne d'Amenemhat $\mathrm{IV}$, dont est datée une stèle découverte dans le temple romain du port de Bérénice ${ }^{39}$.

La localisation de Pount est débattue depuis de longues décennies. Pour le Moyen Empire, on retiendra l'opinion de Rodolfo Fattovich, qui place Pount à la limite du Soudan et de l'Éthiopie, avec un possible lieu de débarquement dans la baie du Ras Aqiq, mais une extension du terme vers les côtes yéménites est envisageable dès cette époque ${ }^{40}$. Est-il raisonnable de penser qu'un naufragé aurait pu dériver depuis le golfe de Suez jusqu'à une île située dans la zone méridionale de la mer Rouge ? Il est clair que non, même si les courants dominants vont vers le sud. Mais on sait que le récit du naufrage (récit 2), empreint de merveilleux, est conçu pour éveiller l'imagination du lecteur. L'Île imaginaire du Souverain de Pount pourra dès lors être aussi lointaine que les dimensions du bateau ou la taille du Serpent sont exagérément grandes ${ }^{41}$.

236.8), tandis que la barque funéraire de Chéops fait 42,30 mètres de long (STEINMANN 2008, p. 251). Voir aussi CANHAO 2012, p. 38-40, 116-118.

37 Voir note 33 ci-dessus pour les références et arguments.

${ }^{38}$ Voir OBSOMER 2019a, p. 40-44, 53-57 ; 2019b, p. 16-20, 36-38.

${ }^{39}$ Références dans OBSOMER 2019b, p. 20-26, 38-39.

${ }^{40}$ Discussion et références dans OBSOMER 2019a, p. 47-49; 2019b, p. 26-28.

${ }^{41}$ Diodore de Sicile (III, 39) et Strabon (XVI.4, 6) rapportent qu'au large du golfe Acathartos, où se trouve le port de Bérénice (Ras Banas), on peut atteindre une île appelée Ophiôdès en raison d'une abondance de serpents. Pour WAINWRIGHT 1946, p. 31-38, il s'agit de St John, en arabe Zeberged. Rien ne permet d'affirmer que cette île était connue des marins qui allèrent vers Pount au Moyen Empire, mais, si c'est le cas, une escale sur celle-ci aurait pu être rapportée par ces marins et inspirer in fine l'auteur du Naufragé. 


\section{b. Le récit 1}

Le récit initial, qui évoque une expédition navale en Nubie, fait référence à une réalité bien attestée au Moyen Empire, proposant un cadre géographique plus strictement véridique. On lit que le bateau dans lequel le šmsw (ex-Naufragé) s'adresse au ḩ`ty- ${ }^{\circ}$ a atteint l'extrémité nord de Ouaouat et franchi l'île de Biggeh (col. 8-10 : ph.n.n phwy W’3’’t, sn.n.n Snmwt). Les deux énoncés, qui présentent chacun une allitération ${ }^{42}$, forment un doublon sémantique. En effet, phwy $W 3 w^{3} t$ ne désigne pas l'extrémité sud de Ouaouat comme Golénischeff le pensait ${ }^{43}$, mais bien son extrémité nord, comme Kurt Sethe fut le premier à l'expliquer ${ }^{44}$. Atteindre le nord de Ouaouat et passer l'île de Biggeh sont une façon d'indiquer la fin imminente du trajet nilotique en $\mathrm{Nubie}^{45}$, même si la frontière officielle de l'Égypte a été déplacée vers la deuxième cataracte durant la XII ${ }^{\mathrm{e}}$ dynastie.

Après une voire plusieurs expéditions sous le règne de Mentouhotep $\mathrm{II}^{46}$, la conquête durable de Ouaouat, entre les première et deuxième cataractes, eut lieu en l'an 29 d'Amenemhat I ${ }^{\text {er }}$ et amena la construction de la forteresse de Bouhen, où des stèles de l'an 5 de Sésostris $\mathrm{I}^{\mathrm{er}}$ ont été trouvées ${ }^{47}$. De nombreuses inscriptions relevées à el-Girgaoui, à mi-chemin entre Éléphantine et Bouhen, attestent une grande activité en Ouaouat dans les années suivantes : on songera à l'administration du territoire conquis, à l'exploitation de ses ressources et à la contruction de nouvelles forteresses ${ }^{48}$. Parmi elles, l'inscription RILN 10A indique que « le chancelier royal Séhétep(ib)rê et le noble prince, chancelier royal et compagnon unique, le courtisan royal véritable qui accomplit tout ce que loue son maître chaque jour, le responsable des bateaux Rédis furent envoyés (en mission) durant 20 ans pour les allées et venues vers Ouaouat du responsable de la ville et vizir (...) Antefoqer $»{ }^{49}$. C'est une fois la présence égyptienne solidement installée à Bouhen, au nord de la deuxième cataracte, que fut menée la première campagne militaire au sud de celle-ci, vers le pays de Kouch (Kerma) ${ }^{50}$. Cette campagne de l'an 17/18 de Sésostris ${ }^{\text {er }}$ amena le roi à se rendre en personne jusqu'à Bouhen, laissant au général Mentouhotep fils d'Âmou (stèle Florence 2540) le soin de diriger en amont les opérations qui allaient donner lieu au versement par les Kouchites d'un tribut, mentionné

42 Par ex. Rendsburg 2000, p. 20 ; Allen 2015, p. 11.

${ }^{43}$ GOLÉNISCHEFF 1906, p. 86-87 ; 1912, p. 46. Voir aussi GoEDICKE 1974, p. 7. Golénischeff pensait que l'expédition du ḩ̉ty- ${ }^{`}$ avait atteint Pount, dont elle était revenue en touchant terre aux « extrêmes limites du pays Ouaoua-t», qu'il situe à Bérénice sur la mer Rouge, après quoi la troupe aurait traversé le désert jusqu'à la première cataracte du Nil (Senmout). Golénischeff allait même jusqu'à envisager que les récits 1 et 2 concernaient la même expédition à Pount, le $h h_{3}{ }^{2}$ - $^{`}$ commandant le bateau qui ramenait le Naufragé en Égypte : voir GOLÉNISCHEFF 1912, p. XII-XX, et la critique justifiée de POSENER 1938, p. 267, n. 2.

${ }^{44}$ SETHe 1907, p. 81. Voir aussi LefebVre 1949, p. 33, n. 6 ; Foster 1992, p. 24 ; 2001, p. 9 ; AlLEN 2015, p. 12. Ainsi, lorsque deux nomes voisins de Haute Égypte offrent le même nom, ils se distinguent par l'ajout de $h n t(y) t$ pour celui qui est en amont, de pht pour celui qui est en aval. C'est le cas des nomes 13 et 14 , des nomes 20 et 21 .

${ }^{45}$ SETHE 1907, p. 81.

${ }^{46}$ POSTEL 2008, p. 339-341.

${ }^{47}$ Stèles EES 882 et Philadelphie E 10995. Texte et références dans OBSOMER 1995, p. 675 et 686.

48 OBSOMER 1995, p. 270-286.

49 ŽÁBA 1974, p. 39-44, fig. 29 ; OBSOMER 1995, p. 648-649.

${ }^{50}$ OBSOMER 1995, p. 311-335. 
dans les Annales d'Amenemhat II. La présence militaire égyptienne sur la deuxième cataracte fut renforcée au cours des règnes suivants, notamment sous Sésostris III, qui établit la frontière sud à Semna et mena en personne plusieurs expéditions vers Kouch ${ }^{51}$.

Le texte du Naufragé ne mentionne pas les objectifs de l'expédition nubienne du ḩ3ty- ${ }^{52}$. Mais cela importe peu : le récit 1 ne fait qu'offrir le cadre spatio-temporel dans lequel sera narré le récit 2 ou récit principal, celui du naufrage. Ce qui compte, c'est l'impression que l'on a que cette mission en Nubie n'a pas rencontré le succès espéré ${ }^{53}$, si bien que le $s m s w$, après avoir prodigué au $h^{3}{ }^{3}{ }^{-}{ }^{-}$des conseils sur l'attitude à avoir lors de la réception royale, est amené à lui raconter « une chose semblable» (mitt iry) qui lui était arrivée précédemment (col. 21-23).

\section{c. Remarques}

1. Pour Claude Vandersleyen, partisan d'une localisation nilotique de Ouadj-our, le naufrage n'aurait pas lieu en mer Rouge, mais sur le cours supérieur du Nil où se trouverait Pount ${ }^{54}$. Parmi les éléments que Christian Cannuyer rassemble comme des arguments en faveur de cette thèse ${ }^{55}$, le premier concerne l'emploi de mitt iry en Naufragé 22 : «L'emploi de mit.t irj pour qualifier la comparaison entre l'expédition évoquée dans le préambule et celle dont le récit va suivre indique entre les deux plus qu'une analogie, une similitude très étroite. Si les deux expéditions avaient eu des contextes aussi différents que l'une le Nil nubien, l'autre la mer Rouge, il est peu vraisemblable que le narrateur se fût exprimé ainsi ».

Il importe de savoir si réellement le šmsw emploie mitt iry dans l'intention de comparer les expéditions navales des récits 1 et 2. Quand le Serpent entreprend de raconter au Naufragé « une chose semblable (mitt iry) qui s'est produite dans cette île » (Naufragé 125), force est de constater qu'il n'est nullement question ici d'expédition navale, mais d'un malheur survenu dans l'île (récit 3) ${ }^{56}$. Il est probable dès lors qu'il en va de même en Naufragé 22, le but du šmsw étant de présenter au $h^{3}{ }^{3} y-{ }^{\complement}$ une situation critique similaire à la sienne, voire plus grave, et d'expliquer ensuite comment il a fini par s'en sortir favorablement ${ }^{57}$. C'est ce que le $\check{s} m s w$ souhaite qu'il advienne au ḩ̉ty- ${ }^{`}$ lorsqu'il sera reçu par le roi, au terme de cette expédition

${ }^{51}$ OBSOMER 2017, p. 1-38.

${ }^{52}$ Aussi ROEDER 2009, p. 91. « A quarrying, mining, or military expedition », selon SIMPSON 1972, p. 51, n. 1 ; 2003, p. 47, n. 1 .

${ }^{53}$ Voir, par ex., De BuCK 1928, p. 69, n. 1 ; BUdGe 1931, p. 88 ; PIEPER 1935, p. 7 ; LefeBVRE 1949, p. 30 ; LiCHTHEIM 1973, p. 211 ; SIMPSON 1972, p. 50 ; MANUELIAN 1992, p. 225 ; PARKINSON 1997, p. 89; CANNUYER 1998, p. 28 ; GALÁN 1998, p. 40 ; 2005, p. 35 ; ALLEN 2015, p. 9 et 13. On notera que pour ROEDER 2009, p. 92-93, rien dans le texte n'indiquerait l'insuccès de l'expédition, mais la peur que le ḩ̉ty- ${ }^{`}$ éprouve lui viendrait seulement de la façon d'aborder la rencontre avec le roi.

${ }^{54}$ Voir notamment VANDERSLEYEN 1990, p. 1022-1024; 1999, p. 77-78.

${ }^{55}$ CANNUYER 2012, p. 176-178; 2019, p. 21.

${ }^{56}$ Il s'agit de la perte de tous les compagnons tant du Serpent que du Naufragé : LEFEBVRE 1949, p. 36, n. 25.

${ }^{57}$ Voir aussi GALÁN 1998, p. 57-58 ; 2005, p. 47-48; WILKINSON 2016, p. 245. CANNUYER 1998, p. 29, résumait d'ailleurs comme suit : "L'histoire présente une série de trois malheurs : le dénouement du troisième, en fait le plus ancien (l'embrasement de l'île), a pour but de relativiser le second (le Naufrage), dont l'issue heureuse doit elle-même apaiser la douleur causée par le premier (l'échec de l'expédition du ḩ̉̇j-`)». 
nubienne qui, peut-on alors déduire, a dû faire face à certains problèmes, que le texte ne semble pas mentionner explicitement ${ }^{58}$.

2. Les avis divergent quant à la date possible de la rédaction du Naufragé durant le Moyen Empire : $\mathrm{XI}^{\mathrm{e}}$ dynastie ${ }^{59}$, début de la XII ${ }^{\mathrm{e}}$ dynastie ${ }^{60}, \mathrm{XX}^{\mathrm{e}}$ siècle avant J.-C. ${ }^{61}$, seconde moitié de la XII ${ }^{\mathrm{e}}$ dynastie $^{62}$. Il en va de même pour la date que l'on attribue à la copie du pErmitage $1115^{63}:$ XI $^{\mathrm{e}}$ dynastie $^{64}$, XII ${ }^{\mathrm{e}}$ dynastie ${ }^{65}$ (milieu ${ }^{66}$, seconde moitié ${ }^{67}$, fin de la dynastie ${ }^{68}$ ).

Au niveau paléographique, une grande similitude a été mise en évidence entre le pErmitage 1115 et le pPrisse ${ }^{69}$, si bien que l'idée qu'ils furent l'œuvre du même copiste a été énoncée ${ }^{70}$ : le pPrisse, qui offre une copie de l'Enseignement de Ptahhotep et de l'Enseignement pour Kagemni, est généralement daté de la seconde moitié de la XII ${ }^{\mathrm{e}}$ dynastie, entre 1900 et 1800 avant J.-C. ${ }^{71}$ Par ailleurs, si on estime que les expéditions navales évoquées dans le Naufragé, en Ouaouat et vers le Sinaï, étaient des activités courantes à l'époque de la rédaction du texte, le règne de Sésostris $\mathrm{I}^{\mathrm{er}}$ constituera un terminus a quo. On pourra même songer à la seconde moitié du règne, car le succès de l'expédition navale envoyée à Pount a pu connaître un certain retentissement à l'époque. Enfin, une analyse comparative du Naufragé et de Sinouhé permet de relever un certain nombre de points communs entre les deux œuvres et d'établir l'antériorité de la rédaction de Sinouhé par rapport à celle du Naufragé ${ }^{72}$.

\section{Texte et traductions de Naufragé 1-11}

Voici le texte des onze premières colonnes du Naufragé, structuré en fonction de la traduction de James P. Allen ${ }^{73}$, reproduite également ci-dessous.

\footnotetext{
${ }^{58}$ Voir néanmoins ce qui sera mis en évidence à la fin de cet article.

${ }^{59}$ SIMPSON 1972, p. 50 ; 1984, col. 619 ; ROEDER 2009 p. 75.

${ }^{60}$ PIEPER 1927, col. 737 ; LefEBVRE 1949, p. 29 ; SIMPSON 1984, col. 619 ; 2003, p. 45 (« somewhat later than 2000 B.C. ») ; HeLCK 1992, p. 73 ; PARKINSON 1997, p. 91 ; ROEDER 2009 p. 75 ; WILKINSON 2016, p. 245.

${ }^{61}$ Foster 1992, p. 24 ; 2001, p. 8 ; ALLEN 2015, p. 9.

62 PARKINSON 1991, p. 115 ; MOERS 1999, p. 52 ; 2001, p. 245.

${ }^{63}$ Cette copie est attribuée au scribe Amen-aâ, fils d'Amény, d'après le colophon (col. 186-189). Voir LENZOMARCHESE 2004, p. 361, pour l'analyse du colophon.

${ }^{64}$ GRIFFITH 1896, p. 5233.

${ }^{65}$ GOLÉNISCHEFF 1913, p. 2 ; SiMPSON 2003, p. 45 ; ROEDER 2009 p. 75.

${ }^{66}$ PARKINSON 1997, p. 91.

${ }^{67}$ WILKINSON 2016, p. 246.

${ }^{68}$ GOEDICKE 1974, p. 1.

${ }^{69}$ VON BOMHARD 1999, p. 51-65, qui confirme l'idée énoncée jadis par GOLÉNISCHEFF 1912, p. I-II, et 1913, p. 2, grâce à une étude comparative portant sur les signes hiératiques et l'agencement du texte. Voir aussi NAGAI 2010, p. 43-54.

${ }^{70}$ GOLÉNISCHEFF 1912, p. II-III ; HODJASH, BERLEV 1997, p. 285-286.

${ }^{71}$ Voir Vernus 2001, p. 55, p. 59 (n. 4-5) ; Ragazzoli 2011, p. 86 ; Hagen 2012, p. 142 ; AlLen 2015, p. 161. La composition du texte est attribuée au début du Moyen Empire par EICHLER 2001, p. 97-107. Voir aussi MATHIEU 2011, p. 61.

${ }^{72}$ Sur l'influence de Sinouhé sur le Naufragé, voir par exemple PosENER 1938, p. 268, n. 9. Une étude sur la question est envisagée.

${ }^{73}$ ALLEN 2015, p. 10.
} 


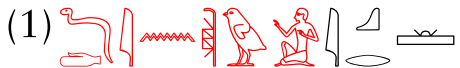

Recitation by an able follower.

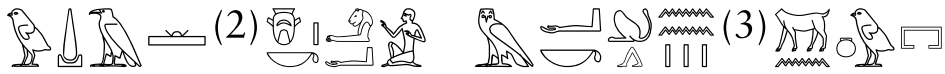

Be informed, high official: look, we have reached home.

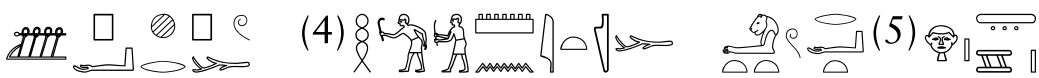

The mallet has been taken, the mooring-post has been hit, and the prow-rope is set on land.

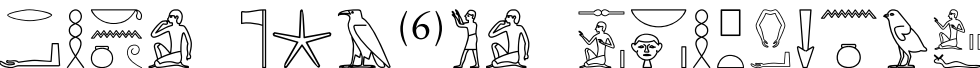

Praise has been given, and thanks, and every man is embracing the other.

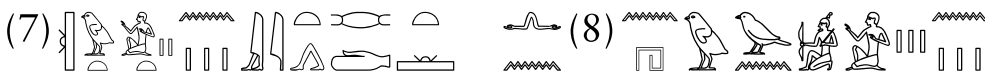

Our crew has returned safe, with no loss of our expedition.

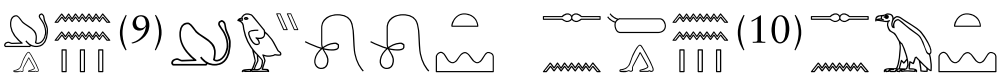

We have reached Wawat's wake, we have gone by Bigga.

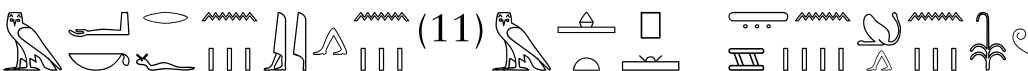

So, look, we have returned in peace; our land, we have reached it.

Les traductions anglaises publiées s'accordent pour la plupart à rendre ph.n.n hnww par «we have reached home $»^{74}$, après que Flinders Petrie et Francis Ll. Griffith eurent proposé «we have come back to the country $»^{75}$, tandis que John L. Foster comprend la phrase comme «we have come through $»^{76}$. En ce qui concerne le terme $\underline{h n w}$, qu'il rend par « home», Allen précise $^{77}$ :

« literally, "the inside": in this case, "inside" Egypt. The term is also used to refer to the capital, Memphis, but the locale mentioned in col. 10 sets the scene in Aswan, first home port for a river expedition returning from south. »

En français, à la suite de Gaston Maspero, on trouve «nous sommes arrivés au pays » ${ }^{78}$, mais « chez nous » chez Pierre Grandet ${ }^{79}$, tandis que Golénischeff avait suggéré « nous avons atteint la patrie ${ }^{80}$. Cette notion de « patrie » pour $\underline{h} n w$ figure dans bon nombre de traductions

${ }^{74}$ BAIKIE 1915, p. 98 ; ViKentieV 1935, p. 24 ; SIMPSON 1972, p. 50 ; 2003, p. 47 ; LiCHTHEIM 1973, p. 212 ; REDFORD 1973, p. 54 ; BRYAN 1979, p. 5 ; PARKINSON 1997, p. 92 ; FOSTER 1988, p. 93 ; RENDSBURG 2000, p. 20 ; LOPRIENO 2003, p. 38 ; QUIRKE 2004, p. 71 ; CASEY 2008, p. 31 ; POE 2008, p. 29 ; ROBERSON 2009, p. 53 ; ABBAS 2013, p. 10 ; ALLEN 2015, p. 10. Voir aussi BudGE 1914, p. 207, « we have arrived at [our] homes »; BETRÒ 1990, p. 32, « siamo arrivati a casa»; BRACAGLION 2007, p. 165, « retornamos ao lar»; CANHAO 2012, p. $13 ; 2013$, p. 93, « nós chegámos a casa ».

75 PETRIE 1895, p. 81 ; 1901, p. 173 ; 1914, p. 41 ; GRIFFITH 1896, p. 5233.

${ }^{76}$ FOSTER 1992, p. $24 ; 2001$, p. 9.

77 ALLEN 2015, p. 11.

${ }^{78}$ MASPero 1905, p. 85 ; 1911, p. 106 ; LefebVRE 1949, p. 32 ; Divin 1957, p. 128 ; Le GuILlOUX 1996, p. 17 ; 2005, p. 19. Voir aussi BRESCIANI 1969, p. 173, « siamo giunti al paese »; CHIOFFI 2005, p. 33, « noi abbiamo raggiunto l'interno (del Paese) ».

79 GRANDET 1998, p. 35.

${ }^{80}$ GOLÉNISCHEFF 1882a, p. $101 ; 1882$ b, p. $341 ; 1891$, p. 177 ; 1906, p. $75 ; 1912$, p. 166 . Voir aussi MASPERO 1882, p. 139 ; LAPIDUS 1995, p. 11. 
allemandes : «wir haben die Heimat erreicht ${ }^{81}$. Mais $\underline{h n w}$ est connu pour désigner la Résidence royale ${ }^{82}$, quelle que soit la période et, par conséquent, sa localisation. C'est pourquoi Adolf Erman traduisit « wir haben die Residenz erreicht ${ }^{83}$, suivi par plusieurs traducteurs en différentes langues ${ }^{84}$. Toutefois, pour cette phrase du début du Naufragé, Erman était loin de songer à la capitale de l'Égypte, soit Licht pour la XII ${ }^{\mathrm{e}}$ dynastie, car il précisait ${ }^{85}$ :

« Der Gefolgsmann weckt seinen Herrn morgens im Schiffe und meldet ihm, daß sie wieder in Ägypten sind; sie haben die Grenzinsel Senmet (heute Bigeh bei Philae) passiert und das Schiff hat schon angelegt. Danach wird mit der Residenz hier Elephantine gemeint sein, also vermutlich die des Fürsten selbst. »

Sethe avait déjà été amené à la même conclusion dès 1907, lorsqu'il observait que la mention du franchissement de la frontière, en l'occurrence Senmout (Biggeh), n'était pas appropriée si les voyageurs se trouvaient déjà à la Résidence royale au milieu de l'Égypte ${ }^{86}$. Il restait alors à prouver soit que le roi avait une résidence sur l'île d'Éléphantine à laquelle le terme $\underline{h} n w$ pouvait s'appliquer sans provoquer de confusion avec la Résidence (Licht), soit, en supposant que le roi résidait chez le $h_{3}^{3} t y-{ }^{`}$, que ce $h{ }^{3} t y-{ }^{`}$ était attaché à la région d'Assouan, ce que le texte n'implique d'aucune manière.

Dans le Wörterbuch der aegyptischen Sprache, le terme hnww de Naufragé 3 est référencé sous l'entrée «Wohnort u.ä. (nicht des Königs) ${ }^{87}$. Plus précisément, c'est l'une des références citées pour justifier l'interprétation «viell. auch im Sinne von: "Heimat" (in die man aus der Fremde zurückkehrt u.ä.) ${ }^{88}$. Les autres textes cités dans les Belegstellen à l'appui de cette interprétation de hnnw comme "Heimat» sont Naufragé 120 et 167, Sinouhé B 94 et 204, Herkhouf (Urk. I, 127.6), sur lesquels nous reviendrons plus loin.

Les auteurs des traductions mentionnées ci-dessus, aussi variées qu'elles furent, semblent s'être accordés à penser que c'est à Assouan, voire au quai de l'île d'Éléphantine, que se trouve amarré le bateau du $h_{3}{ }^{2} y{ }^{-}{ }^{`}$ et de son $s_{m s w}$ au retour de leur expédition nubienne ${ }^{89}$, ce qui suppose la présence du roi à Éléphantine au même moment. Mais dans ses dernières publications, Günter Burkard propose la traduction « wir haben die Hauptstadt erreicht » ${ }^{90}$, sans

\footnotetext{
${ }^{81}$ RoEder 1927, p. 17 ; SCHOtT 1950, p. 170 ; BrunNER-Traut 1963, p. 5 ; 1990, p. 34 ; GoEdiCKE 1974, p. 72 ; MODE 1980, p. 9 ; BURKARD 1983, p. 112. Voir aussi WiEDEMANN 1906, p. 25, « wir erreichten das Heimatland»; DE BUCK 1928, p. 69, «wij hebben het vaderland bereikt ». Par contre, WIEDER 2007, p. 29, n. 88, traduit « wir haben das Innere erreicht».

${ }^{82} \mathrm{~Wb}$. III, 370, 5-10.

${ }^{83}$ ERMAN 1906, p. 5 ; 1923, p. 57.

${ }^{84}$ PiePer 1935, p. 7 ; KASter 1968, p. 282 ; LAlOUetTe 1987, p. 153 ; Hodel-Hoenes 1990, p. 130 ; GAlÁN 1998, p. 35 ; 2005, p. 31 ; Hilbig 2012, p. 39 ; StAuder 2013, p. 155 ; DessoudeiX 2016, p. 28 ; WILKINSON 2016, p. 246. ROEDER 2009, p. 93, traduit d'une part « Residenz », d'autre part « Heimat ».

${ }^{85}$ ERMAN 1923, p. 57, n. 2. Les italiques sont miennes. Traduction anglaise : ERMAN 1927, p. 30, n. 1.

${ }^{86}$ SETHE 1907, p. 81.

${ }^{87} \mathrm{~Wb}$. III, 370, 2-4.

${ }^{88} \mathrm{~Wb}$. III, 370, 2.

${ }^{89}$ Par ex. SIMPSON 1984, col. 619 : « The opening scene is evidently set in Elephantine, since the expedition has reached the limits of Wawat and passed the island of Senmet (Bigga) ».

${ }^{90}$ Burkard 1993, p. 108 ; 2003, p. 142-143. Précédemment, Budge 1931, p. 88, avait traduit « we have reached the Court ».
} 
toutefois en détailler les implications. Les pages qui suivent vont démontrer que Burkard a raison et que c'est au port de Licht que le bateau du hỉty- ${ }^{`}$ se trouve amarré avant la réception de celui-ci au palais royal de Licht.

\section{Le terme hnw et sa graphie}

Le texte du Naufragé utilise le terme $\underline{h n w}$ à quatorze reprises ${ }^{91}$ : sept fois dans la préposition composée $m$ - $h n w$ "à l'intérieur (de)», sept fois en tant que substantif. Invariablement, sa graphie présente le signe $\square$ (O 1) comme déterminatif/classificateur.

Les occurrences de la préposition composée, qui intéressent finalement peu notre propos, sont les suivantes ${ }^{92}$ : (col. 43) S $\underline{d} r . k w i$ m- $\underline{h} n w$ w $n(y) k \jmath p n(y) h t$ « Je me suis couché à l'intérieur d'un abri de bois »; (col. 52 et 115) Nn ntt nn st m-hnww.f « Il n'y a rien qui ne se trouve en elle

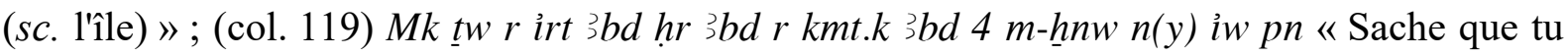
passeras mois après mois jusqu'à ce que tu totalises 4 mois dans cette île »; (1.156) " $h^{c} . n d p t$ tf ii.t(i) (...), sỉ.n.i ntyw m-hnw.s « Alors ce bateau est venu (...) et j'ai reconnu ceux qui étaient à son bord»; (1. 169) Mḥ.k kni.k $m$ hrdw.k, rnpy.k m-hnnw krst.k « Tu empliras tes bras de tes enfants, tu rajeuniras à l'intérieur de ta sépulture ${ }^{93}$; (1. 175) ỉnw pn inn(w).n.ỉ m-hnnw $n(y)$ îw $p n$ « les produits que j'avais rapportés de cette île » (la préposition marque ici l'origine).

Les six mentions du substantif hnnw qui s'ajoutent à la mention initiale de la colonne 3 figurent dans les quatre passages suivants. La traduction «Résidence» a été appliquée d'emblée ${ }^{94}$.

- (col. 120-122) 'Iw dpt $r$ ilt $m$ h hnw, skdw im.s rhh(w).n.k. Šm.k hnn'sn $r \underline{h n w}$, mt.k m niwt.k «Un bateau viendra de la Résidence, ayant à son bord des matelots que tu connais. Tu partiras avec eux vers la Résidence et tu mourras dans ta ville »;

- (1. 135) Ph.k hnww, wn.k im.f $m-k \jmath b n(y) s n w . k$ «Tu gagneras la Résidence, de sorte que tu y sois au milieu de tes frères et sœurs »;

- (1. 167) Mk tw r spr $r \underline{h n w} n$ 3̉bd 2 «Sache que tu parviendras à la Résidence en deux mois ") ;

- (1. 173) $N^{c} t p w i r(w) . n . n m$ hd $r \underline{h n w} n(y)$ ity, spr.n.n $r \underline{h n w}$ ḥr 3 bd 2 « C'est naviguer vers le nord que nous fîmes vers la Résidence du souverain, et nous arrivâmes à la Résidence en deux mois ».

Ces six attestations figurent toutes dans le récit du naufrage (récit 2). Dans les trois premiers passages, le Serpent annonce au Naufragé son départ prochain de l'Île et son retour dans sa ville. Le quatrième précise que cela s'est bien réalisé. Les traducteurs s'accordent à penser que $h n w n(y)$ ity (1.173) désigne la «Résidence du souverain », à savoir Licht pour la

\footnotetext{
${ }^{91}$ Voir aussi ROBERSON 2009.

92 On notera l'usage à trois reprises du nisbé $n(y)$ devant le substantif.

${ }^{93}$ Voir RoBERSON 2009, qui plaide en faveur d'une préposition composée (p. 55 : «You will become young inside tour burial »), en accord avec la traduction de ERMAN 1906, p. 22.

${ }^{94}$ Ainsi procèdent également ERMAN 1906, p. 15, 17, 22 ; 1923, p. 60-62 ; 1927, p. 32-34 ; BUDGE 1931, p. $92-94$ (qui traduit « the Court ») ; KASTER 1968, p. 285-286; LALOUETTE 1987, p. 155-157 (qui omet les occurrences de la 1. 173) ; GALÁN 1998, p. 37-39; 2005, p. 32-33 ; BOMMAS 2003, p. 43 ; WILKINSON 2016, p. 248-249.
} 
XII ${ }^{\mathrm{e}}$ dynastie, mais nombreux sont ceux qui appliquent aux autres mentions de $h n w$ la traduction différente qu'ils avaient choisie pour la mention initiale de la colonne 3 (récit 1), au point que $h n w n(y)$ ity leur semble devoir constituer une exception ${ }^{95}$. Tel n'est pas l'avis exprimé dès 1938 par Georges Posener ${ }^{96}$, qui voyait en chaque occurrence du terme $h n w$ du récit 2 une désignation de la résidence royale de Licht. En effet, la ligne 173 mentionne un trajet de deux mois vers la Résidence de Licht, qui correspond au trajet de deux mois annoncé par le Serpent à la ligne 167 pour se rendre vers $\underline{h n} w$, qui, par conséquent, doit être Licht. Il en va de même à la colonne 122 et à la ligne 135, où hnww est renseigné comme la destination finale du trajet retour, l'endroit où le Naufragé pourra revenir dans sa ville, là où sa famille réside. Dans ce cas, il semble que, dans la colonne 120, le point de départ de l'expédition qui viendra le chercher soit également la Résidence royale de Licht.

Avant d'examiner la question des itinéraires que cette interpétation du terme $h n w$ implique, d'une part dans le récit du naufrage (récit 2), d'autre part dans le récit-cadre (récit 1), il convient de confirmer l'usage de la graphie désigner la Résidence royale. Dans le papyrus R de Sinouhé (pBerlin 10499), attribué à la XIII ${ }^{\mathrm{e}}$ dynastie, les trois occurences conservées de $\underline{h} n w$ offrent le déterminatif $(\mathrm{R} 8,30,59)$, alors que le papyrus $\mathrm{B}$ ( $\mathrm{pBerlin} 3022$ ), qui date sans doute du règne d'Amenemhat III, atteste une seule fois $h n w$ avec le déterminatif $(\mathrm{B} 6)^{97}$, mais sept fois avec le déterminatif $\square$ (B 36, 94, 150, 157, 188, 204, 232). Dans la phrase d'Âmmounenchi « S'est-il passé quelque chose à la Résidence ? », on constate que hnww présente le déterminatif en R 59, mais le déterminatif $\square$ en $B 36^{98}$; la réponse de Sinouhé évoque le décès du roi Amenemhat ${ }^{\text {er }}$, dont on sait par ailleurs qu'il eut lieu dans les appartements privés (' $h n w t y)$ du palais royal. Si le Wörterbuch préconise de comprendre hnww comme «Heimat» en B 94 et $204^{99}$, on ne peut exclure la traduction de $h n w$ par «Résidence», d'autant que B 204 évoque l'enterrement de Sinouhé et que la tombe de ce dernier est située à Licht par la fin du texte. En B 188 et 243, il est clair que $\underline{h n w}$ désigne la Résidence royale ${ }^{100}$. Les textes épigraphiques des règnes de Sésostris $I^{\text {er }}$ et d'Amenemhat II font également usage, pour désigner la Résidence royale, d'une graphie de $\underline{h n w}$ avec le déterminatif $\square$, comme on l'observe dans les inscriptions des tombes d'Amény à Béni Hassan ${ }^{101}$ et de Sarenpout $\mathrm{I}^{\mathrm{er}}$ à Assouan ${ }^{102}$, et sur les stèles d'Oupouaout-âa à

${ }^{95}$ Ainsi LeFEBVRE 1949, p. 32, n. 5 : « Le mot hnw sera partout, dans ce conte, traduit « pays » (home), sauf 1. 173, où l'expression hnww $n$ ity désigne la résidence royale ». Pour éviter cette construction génitivale, Galán et Allen font de $r$ hnw $n$ ity deux compléments prépositionnels distincts : GALÁN 1998, p. 39, n. 45, " hacia la Residencia... por el soberano »; GALÁn 2005, p. 33, n. 45, « to the Residence... on the Sovereign's behalf »; ALLEN 2015, p. 48-49, « to home and the sire ».

96 POSENER 1938, p. 268, n. 1.

${ }^{97} \mathrm{KoCH} 1990$ p. 13 : «Je n'ai pas songé à me rendre à cette Résidence » (B $6=\mathrm{R}$ 30), lorsque Sinouhé se décide à fuir plutôt que de gagner Licht.

${ }^{98}$ KOCH 1990 p. 26.

${ }^{99} \mathrm{Ci}$-dessus, note 88.

100 Sinouhé B 188-189 : «Puisses-tu (re)voir la Résidence où tu as grandi, baiser le sol à la grande double porte, et te joindre aux compagnons ». En B 243, le commandant de la patrouille frontalière envoie à la Résidence un message pour faire savoir au roi que Sinouhé se trouve à la frontière.

${ }^{101}$ Urk. VII, 21.1 : le nomarque est quelqu'un « que la qenbet de la Résidence appréciait ». 
Abydos (Leyde V 4 et Munich Gl. WAF 35) ${ }^{103}$. C'était déjà le cas sous la $\mathrm{VI}^{\mathrm{e}}$ dynastie, notamment dans les inscriptions d'Herkhouf et de Pépinakht à Assouan, lorsque ces derniers évoquaient leur retour à la Résidence royale de Memphis au terme de leurs missions en Nubie $^{104}$.

En conclusion, l'absence du signe de la ville dans les sept occurrences du substantif $\underline{h} n w$ qui figurent dans le Naufragé n'interdit pas de penser à la Résidence royale dans chaque cas, tout en confirmant l'attribution à la XII ${ }^{\mathrm{e}}$ dynastie de la copie du pErmitage 1115 .

\section{Les itinéraires des expéditions navales}

Dans le récit principal (récit 2), trois trajets sont mentionnés successivement : (a) le trajet aller du Naufragé avant la tempête ; (b1) le trajet aller du bateau qui viendra le chercher sur l'Île ; (b2) le trajet retour de ce même bateau.

(a) Le trajet aller du Naufragé est exprimé à deux reprises. En Naufragé 23-25: «J'étais

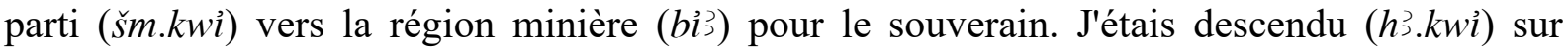
Ouadj-our dans un bateau (...) ». En Naufragé 89-91 : "J'étais descendu ( $h$ ว.kwi) vers la région minière $(b i 3)$ en mission du souverain dans un bateau (...)». Si $\breve{s}$ est un verbe de mouvement très général, le verbe $h_{3} i$ est bien connu lorsqu'il s'agit d'embarquer sur un bateau $^{105}$. Compte tenu de l'identification de $b i 3$ au Sinaï préconisée ci-dessus, le franchissement de la mer Rouge au départ d'un port intermittent est impliqué ${ }^{106}$, dans le cadre d'une mission qui, nécessairement, inclura deux trajets à pied.

(b1) Le trajet aller de ceux qui sauveront le Naufragé est annoncé comme suit par le Serpent : "Un bateau viendra de la Résidence ('Iw dpt $r$ lit $m \underline{h n w}$ ), ayant à son bord des matelots que tu connais » (Naufragé 119-120). La formulation sera examinée ci-après.

(b2) Leur trajet retour est évoqué dans trois passages : «Tu partiras ( $\check{m} m . k)$ avec eux vers la Résidence » (Naufragé 122) ; "Sache que tu parviendras (Mk tw r spr) à la Résidence en deux mois » (Naufragé 167-168) ; «C'est naviguer vers le nord ( $n^{`} t m$ hd) que nous fîmes vers

\footnotetext{
102 Inscription de la porte d'entrée (GARDINER 1908, pl. VI, col. 11 ; Urk. VII, 3.4) : « Sa Majesté [a fait que] je me déplace en toute liberté comme tout magistrat $(s r)$ de sa Résidence ». Mais notation avec le déterminatif de la ville dans la copie peinte à l'intérieur de la tombe (GARDINER 1908, pl. VII, col. 11 ; Urk. VII, 3.5).

${ }^{103}$ ANOC 20.1-2. Oupouaouat-âa évoque sa rencontre avec le roi. Leyde V 4, ligne 8 : «Je siégeai à ma place dans le temple, après être allé vers le nord et être revenu de la Résidence vers le sud en étant informé » (BOESER 1909, pl. IV). Munich Gl. WAF 35, ligne 15 : «J'ai navigué vers le nord pour rendre l'hommage en cette grande Résidence de sa Majesté » (DYROFF, PÖRTNER 1904, pl. II, n 3 ).

${ }^{104}$ Herkhouf, expédition 3 : « la troupe de Iam qui revenait avec moi vers la Résidence, en compagnie de la troupe envoyée avec moi » (Urk. I, 127.6) ; «lorsque le serviteur que je suis voyageait donc vers la Résidence, on fit que vienne à ma rencontre (...) Khouni, avec des bateaux chargés de vin de dattes, de gâteaux, de pain et de bière » (Urk. I, 127.12). Copie de la lettre de Pépi II : «Toi, viens immédiatement vers le nord, vers la Résidence !» (Urk. I, 129.15); "Si tu parviens à la Résidence tandis que ce nain est avec toi en vie et en bonne santé, alors ma Majesté accomplira pour toi des choses plus grandes que ce qui fut fait au chancelier du dieu Baourdjed» (Urk. I, 130.16). Pépinakht, première mission : «J'en ai ramené un grand nombre à la Résidence comme prisonniers » (Urk. I, 133.14). Deuxième mission: «J'ai ramené les souverains de ces pays à la Résidence en paix, ainsi que du gros et petit bétail vivant » (Urk. I, 134.6).

${ }^{105} \mathrm{~Wb}$. II, 472, 9-13.

106 Voir aussi ALLEN 2015, p. 16.
} 
la Résidence du souverain ( $r \underline{h n w}$ ), et nous arrivâmes (spr.n.n) à la Résidence en deux mois » (Naufragé 172-173). Si un verbe de mouvement général (šm ou spr) est employé à trois reprises, l'emploi du verbe $n^{` i}$ en Naufragé 172 nécessite lui aussi un examen plus pointu.

Le texte mentionne donc une navigation entre la Résidence de Licht et l'Île du Serpent, tant à l'aller (Naufragé 119-120) qu'au retour (Naufragé 172-173). Dans son étude de 1988, Bradbury supposait un trajet empruntant le canal du Nil à la mer Rouge, qui aurait été creusé par le Sésostris des textes classiques ( $c f r$ Aristote, Strabon et Pline) et qui, dès le règne de Sésostris III, aurait rendu obsolète l'usage du port de $S_{3}^{3} w w$, localisé à Mersa Gaouasis en 1976 par Abdel Monem Sayed ${ }^{107}$. Mais cette idée de Bradbury est caduque, à la fois parce que les fouilles menées à Mersa Gaouasis entre 2001 et 2011 ont livré plusieurs documents datés des successeurs de Sésostris III, Amenemhat III et IV $^{108}$, et parce que les trois auteurs classiques qui attribuent à Sésostris le projet de creuser le canal du Nil à la mer Rouge affirment aussi que ce roi dut renoncer à mener à bien ce projet ${ }^{109}$. Hérodote, qui mentionna ce canal bien avant Aristote, attribue le projet à Nécôs (Nékao II) et son achèvement à Darius I ${ }^{\mathrm{er} 110}$, ce que confirment les stèles de Darius dressées sur le parcours du canal et publiées par Posener ${ }^{111}$. Dès lors qu'un trajet entièrement naval entre la Résidence de Licht et l'Île du Serpent semble exclu, il convient à mon sens de suivre ici aussi l'avis de Posener, pour qui l'auteur du Naufragé «a très bien pu abréger les descriptions des voyages qui avaient peu d'importance pour son histoire dont la partie principale se place dans l'île du serpent. Il aurait ainsi passé sous silence la traversée du désert et donné au lecteur l'impression que les navires passaient directement du fleuve à la mer et vice versa ${ }^{112}$. Bref, l'équipage peut venir de Licht et embarquer sur la côte de la mer Rouge pour effectuer le trajet qui sauvera le Naufragé (Naufragé, 119-120). Quant au retour (Naufragé, 172-173), si le verbe $n^{` i}$ est employé pour la

${ }^{107}$ BRADBURY 1988, p. 144, n. 87. Extraits significatifs : « It is noteworthy that with Sesostris III the naval traffic dispatched from $S w w$ ceased altogether. Importantly, Strabo (Geography, 1.2.31; 17.1.25), Pliny (Natural History, VI, xxxiii: 165), and Aristotle (Météorologie, 352, b 26) reported that a Sesostris cut a canal from the Nile to the Red Sea, an endeavor which could account for the abandonment of $S w w$ during Sesostris III's reign "; "Since the Shipwrecked Sailor's story has been cited as implying the use of this waterway, or even having been formulated because of the opening of the canal (...), the tale most likely represents the trading practice at the close of the 12th Dynasty. The Delta Canal seems to have been navigable when Hatshepsut's fleet sailed to Punt $»$.

108 Voir en dernier lieu BARD, FATTOVICH 2018, p. 26-27 et 62-89. Sayed avait plaidé contre l'existence d'un canal du Nil à la mer Rouge sous la XII ${ }^{\mathrm{e}}$ dynastie : SAYED 1983, p. 35-36 ; 2003, p. 433 ; 2006, p. 207-208.

${ }^{109}$ Aristote, Météor. I, 14, 27 (352b) : « Un de leurs rois essaya de le creuser. (...) On dit que Sésostris fut le premier des (rois) anciens à l'entreprendre. Mais il trouva que la mer était plus haute que la terre. C'est pourquoi il mit un terme au creusement, lui d'abord et Darius ensuite ». Voir aussi Strabon, Géogr., XVII, 1, 25 (804), et Pline, Hist. nat., VI, 33 (165).

${ }^{110}$ Hérodote, Hist., II, 158. Voir aussi Diodore, Bibl. hist., I, 33. Il est probable qu'Aristote attribua ce canal à Sésostris parce qu'on lisait chez Hérodote (II, 108) que Sésostris avait fait creuser tous les canaux d'Égypte par les captifs ramenés des pays conquis : OBSOMER 1989, p. 40.

${ }^{111}$ Posener 1936, p. 48-87, pl. IV-XV. Voir aussi VANDERSTEEN 2018, p. 57-117, pour une traduction des textes en différentes langues.

112 Posener 1938, p. 269. Voir aussi ERMAN 1923, p. 60, n. 2 ; 1927, p. 32, n. 3. Ce processus s'observe d'ailleurs aussi dans un récit de faits réels comme le récit des voyages d'Herkhouf vers Iam, où seuls les trajets en caravane sont détaillés, tandis que les déplacements en bateau sur le Nil sont omis ou à peine évoqués : voir OBSOMER 2007, p. 46-50 et fig. 3. 
navigation vers le nord, le verbe $s p r$ complète la description du trajet aboutissant effectivement à la Résidence.

Dans le récit-cadre (récit 1), les données relatives au franchissement de la première cataracte du Nil, au retour de l'expédition nubienne, sont exprimées par deux propositions verbales attestant une forme sdm.n.f: ph.n.n phwy $W^{3} w^{3}$ 't, sn.n.n Snmwt. Alors qu'on trouvait Mk ph.n.n à la colonne 2, l'absence de particule $m k$ (ou d'auxiliaire) invite à ne pas y voir deux propositions autonomes. L'absence d'élément circonstanciel ne permet pas non plus de les considérer comme des formes verbales en fonction emphatique. Comme il est clair qu'il ne s'agit pas de séquentielles, on choisira d'y voir deux circonstancielles, soit : " après que nous avons atteint le nord de Ouaouat et franchi Senmout ${ }^{113}$. Ceci permet de limiter au cours égyptien du Nil la mention de l'absence de problèmes pour l'équipage et de suggérer qu'il n'en avait pas été de même lors de la mission en Nubie ${ }^{114}$. De là les craintes du chef de l'expédition, le ḩ̉ty-c, peu avant d'être reçu en audience par le roi et la volonté du šmsw de mettre en évidence le soulagement de ceux qui sont rentrés à bon port. Par conséquent, le terme $\underline{h n} w$ de l'énoncé initial Mk ph.n.n hnww désigne bien la Résidence de Licht au port de laquelle le bateau est amarré ${ }^{115}$.

Il convient enfin d'expliquer l'énoncé final (proposition autonome avec complément direct antéposé) : T3.n, ph.n sw « Notre pays, nous l'avons atteint » (perfectif) ou « Notre pays, nous allons l'atteindre » (prospectif). Le terme $t$ ? désigne-t-il l'Égypte dans son ensemble ou la région dont les marins sont originaires ? On notera, d'une part, que dès le règne de Sésostris $\mathrm{I}^{\mathrm{er}}$, la frontière sud de l'Égypte avait été fixée bien au sud d'Assouan, aux abords de la deuxième cataracte du Nil. D'autre part, une stèle privée d'Akhmim du début de la XII ${ }^{\mathrm{e}}$ dynastie atteste

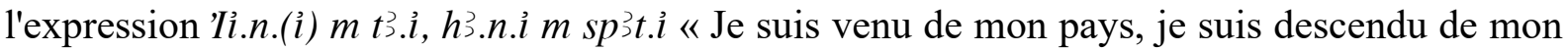
nome », avec $t 3 . \hat{l}$ « mon pays » qui remplace le terme habituel niwt. $\dot{l}$ « ma ville » ${ }^{116}$. Comme on sait grâce au récit du naufrage que le $\breve{s} m s w$ était originaire de $\mathrm{Licht}^{117}$, il est raisonnable de penser que $t 3 . n$ "notre pays" désigne la ville de Licht et sa région à la colonne $11 \mathrm{du}$ Naufragé. La phrase indique dès lors que les marins sont rentrés chez eux ou vont pouvoir rentrer chez eux sous peu. Dans la traduction qui suit, j'ai choisi le prospectif, suggéré par mon collègue Bernard Mathieu ${ }^{118}$.

${ }^{113}$ Voir aussi ERMAN 1906, p. 5. Certains traduisent par des propositions circonstancielles, mais en les faisant dépendre de ce qui suit et non de ce qui précède (SIMPSON 1972, p. 51 ; VANDESLEYEN 1990, p. 1021), ou font de la première proposition une circonstancielle et de la seconde une principale (BURKARDT 1993, p. 46).

${ }^{114}$ Voir, ci-après, la traduction intégrale du passage étudié.

115 Ce port est mentionné dans Sinouhé B 247.

${ }^{116}$ Caire CG 20024, ligne 8 : WiLdUng 1984, p. 11, fig. 1. Voir aussi JANSSEN 1946, II A 21 ; Wb. V, 215.7.

${ }^{117}$ Notamment Naufragé 132-135 : « Si tu as du courage et (si) ton cœur est ferme, (alors) tu empliras tes bras de tes enfants, tu embrasseras ta femme et tu verras ta demeure. Cela est bon plus que tout (autre) chose. Tu gagneras la Résidence, de sorte que tu y sois au milieu de tes frères et sœurs "). Voir aussi Naufragé 122, 158159, 167-169.

${ }^{118}$ Communication du 15 février 2020. 


\section{Traduction proposée au terme de cette étude}

Voici la traduction proposée des onze premières colonnes du texte du Naufragé :

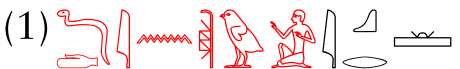

Dd in ${ }^{119}$ šmsw ikr :

Dire par l'excellent chemsou :

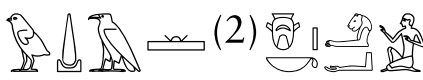

$W \underline{d}$ ’ ib.k, histy- ${ }^{c}$.

Sois informé ${ }^{120}$, prince.

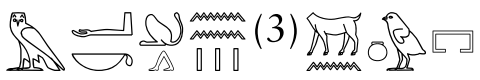

Mk ph.n.n hnww.

Sache que nous avons atteint la Résidence.

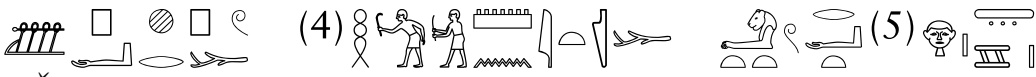

Šsp(w) hrpw, hww(w) mnit, ḩ̉tt rdi.t(i) hr t’3.

Le maillet a été saisi et le poteau a été frappé, après que l'amarre a été placée par terre.

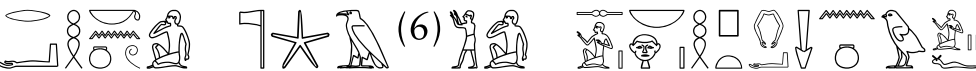

$R \operatorname{di}(w) h k n w, d w 3(w) n t r, s$ nb hr hpt snnw.f,

Une prière a été faite et le dieu a été remercié, chacun embrassant son compagnon,

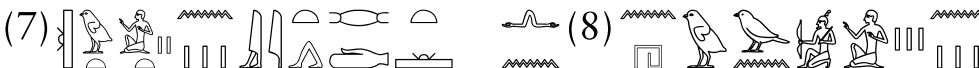

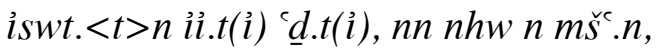

(car) notre équipage est revenu en bonne santé sans aucune perte pour notre troupe,

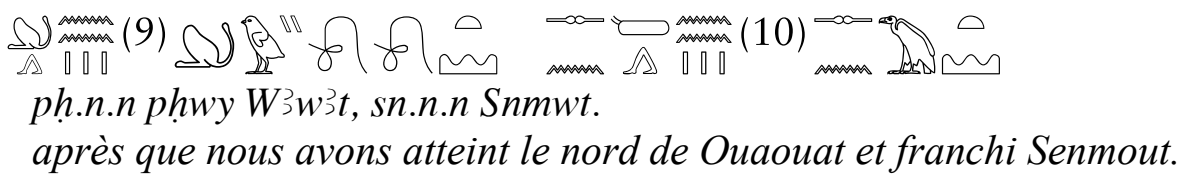

Dom

Mkrfn il.(wy)n m htp.

Nous voici revenus en paix.

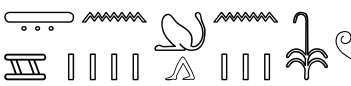

T3.n, ph.n sw.

Notre pays, nous allons l'atteindre.

${ }^{119} \underline{D} d$ in šmsw est noté à l'encre rouge. Infinitif suivi d'un complément d'agent introduit par in, comme suggéré par BAINES 1990, p. 58, malgré les doutes exprimés par BOLSHAKOV 1993, p. 254-259. Voir aussi ABBAS 2013, p. 10 ; ALLEN 2015, p. 11.

${ }^{120}$ SETHE 1907, p. 80 ; Wb. I, 400.11. Voir aussi ABBAS 2013, p. 10 ; ALLEN 2015, p. 10. La forme causative $s w d$ ¿ $i b$ « informer » est très bien attestée dans les lettres : $W b$. IV, 80.6-13. En somme, la phrase indique que le šmsw fait son rapport au ḩ̉ty-` suite aux manœuvres d'accostage qu'il a dû superviser. Notons que DE BUCK 1928, p. 69, rendait $w \underline{d}$ ’ ib. $k$ par « Goed nieuws ». 


\section{CARTE}

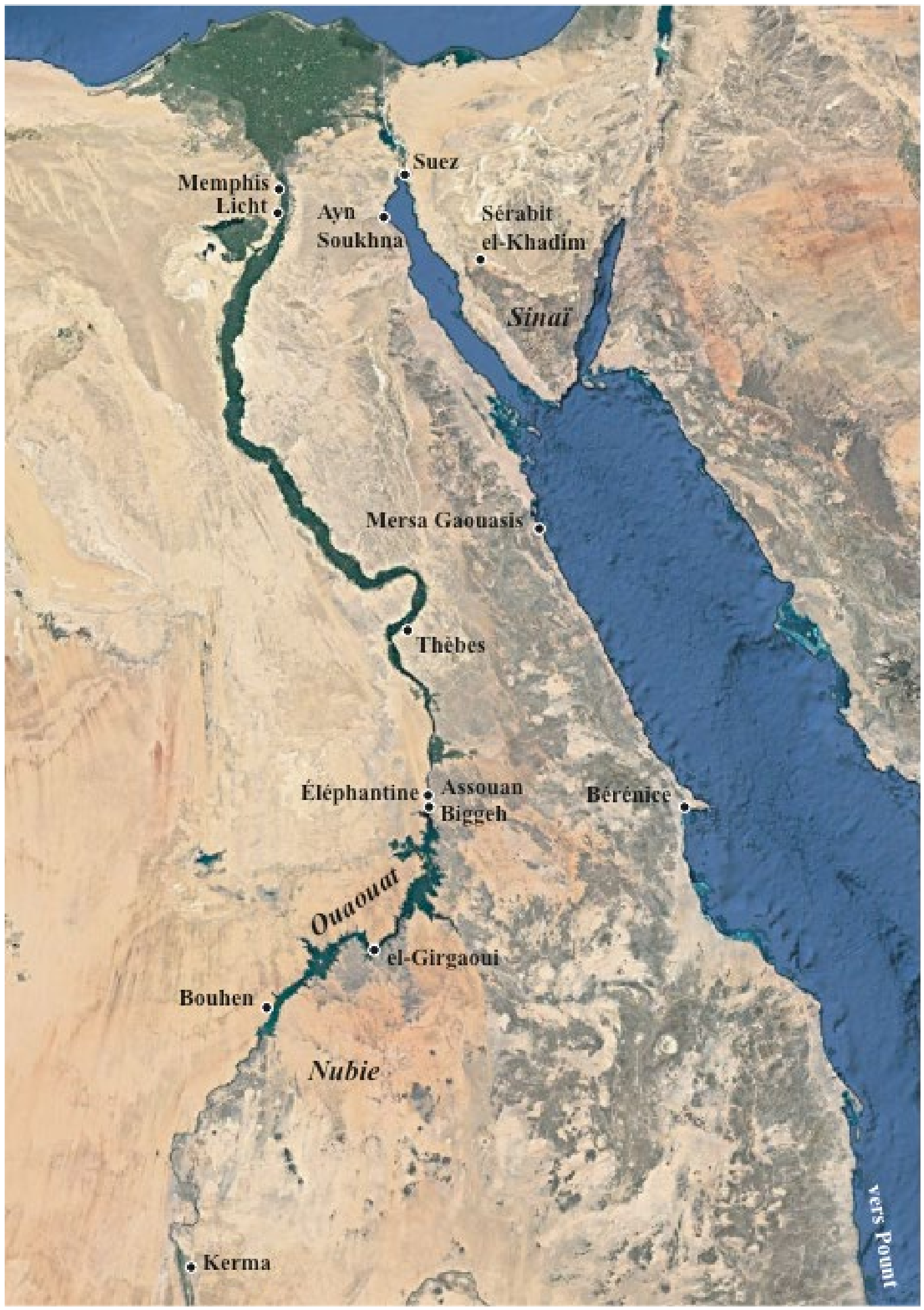




\section{BIBLIOGRAPHIE}

ABbAS Eltayeb, 2013 : "The Tale of the Shipwrecked Sailor and the Rite of Passage ", Journal of the American Research Center in Egypt, 49, p. 9-18.

AbD el-Raziq Mahmoud, Castel Georges, Tallet Pierre, GhicA Victor, 2002 : Les inscriptions d'Ayn Soukhna (Mémoires publiés par les membres de l'IFAO, 122), Le Caire.

Allen James P., 2015 : Middle Egyptian Literature. Eight Literary Works of the Middle Kingdom, Cambridge.

BAIKIE James, 1915 : Wonder Tales of the Ancient World, Londres.

BAINES John, 1990 : « Interpreting the Story of the Shipwrecked Sailor », Journal of Egyptian Archaeology, 76, p. 55-72.

BARD Kathryn A., FAtTovich Rodolfo, 2018 : Seafaring expeditions to Punt in the Middle Kingdom: Excavations at Mersa/Wadi Gawasis, Egypt (Culture and History of the Ancient Near East, 96), Leyde, Boston.

Betrò Maria Carmela, 1990 : Racconti di viaggio e di avventura dell'antico Egitto (Testi del Vicino Oriente antico, I. Letteratura egiziana classica, I), Brescia.

Blackman Aylward M., 1932: Middle-Egyptian stories (Bibliotheca Aegyptiaca, 2), Bruxelles.

Boeser Peter Adrian Aart, 1909 : Beschreibung der aegyptischen Sammlung des niederländischen Reichsmuseum der Altertümer in Leiden, II, 1, La Haye.

BolshaKov Andrey O., 1993 : « Some de visu Observations on P. Hermitage $1115 »$, Journal of Egyptian Archaeology, 79, p. 254-259.

Bommas Martin, 2003: «Heimweh nach Theben vor dem Hintergrund kultureller Lebensform », Göttinger Miszellen, 193, p. 41-44.

BRADBURY Louise, 1988: "Reflections on Travelling to "God's Land" and Punt in the Middle Kingdom », Journal of the American Research Center in Egypt, 25, p. 127-156.

Brancaglion Antonio, 2007 : «O Conto do Náufrago: Papiro Ermitage 1115 », Tiraz, 3, p. 161-191.

BRESCIANI Edda, 1969 : Letteratura e poesia dell'antico Egitto, Turin.

BREASTED James Henry, 1906 : Ancient Records of Egypt, vol. I, Chicago.

BRUNNER-TRAUT Emma, 1963 : Altägyptische Märchen, Düsseldorf, Cologne.

_, 1990 : Altägyptische Märchen, Mythen und andere volkstümliche Erzählungen, $8^{\mathrm{e}}$ éd., Munich.

BRYAN Betsy M., 1979 : «The Hero of the "Shipwrecked Sailor” », Serapis, 5, p. 3-13.

Budge Ernest Alfred Wallis, 1914 : The Literature of the Ancient Egyptians, Londres.

—, 1931 : Egyptian Tales and Romances, Londres.

BURKARD Günter, 1983 : «Der formale Aufbau altägyptischer Literaturwerke », Studien zur altägyptischen Kultur, 10, p. 79-118. 
—, 1993 : Überlegungen zur Form der ägyptischen Literatur: Die Geschichte des Schiffbrüchigen als literarisches Kunstwerk (Ägypten und Altes Testament, 22), Wiesbaden.

BURKARD Günter, THISSEN Heinz-Josef, 2003 : Einführung in die altägyptische Literaturgeschichte, 1. Altes und mittleres Reich, Hambourg, Londres.

Canhao Telo Ferreira, 2012: O Conto do Náufrago. Um olhar sobre o Império Médio Egípcio. Análise histórico-filológica / The Tale of the Shipwrecked Sailor. A Glance over Egypt's Middle Kingdom. A Historical-Philological Analysis, Lisbonne.

—, 2013 : Doze textos egípcios do Império Médio. Traduçoes integrais, Coimbra.

CANNUYER Christian, 1998: «Le voyage comme tension eschatologique dans l'Égypte ancienne. Les leçons du Naufragé », dans P. NAster, J. Ries, A. VAN TONGerloo (éd.), Les voyages dans les civilisations orientales (Acta Orientalia Belgica, 11), Bruxelles, Louvain-la-Neuve, Leuven, p. 27-42.

—, 2012 : "Tempête en Nil! Nouvelle note sur le grain qui fit sombrer le navire du Naufragé », dans C. CANnuYer, D. De Smet, R. Lebrun (éd.), Regards sur l'Orientalisme belge: suivis d'études égyptologiques et orientales. Mélanges offerts à Claude Vandersleyen (Acta Orientalia Belgica, 25), Bruxelles, p. 175-184.

—, 2019 : «ouadj-our, "le grand vert" pour les nuls. Bref regard sur une controverse égyptologique », dans C. CANNUYER, La mer, les ports, les marins dans les civilisations orientales. Pauline Voûte et Robert Donceel in honorem (Acta Orientalia Belgica, 32), Bruxelles, p. 15-30.

CASEY Christian, 2008 : The Story of the Shipwrecked Sailor, Austin.

Chioffi Marco E., Le Guilloux Patrice, 2005 : Il Racconto del Naufrago / Le Conte du Naufragé, Milan.

DE BuCK Adriaan, 1928 : Egyptische Verhalen uit het oud-Egyptisch vertaald, Santpoort.

—, 1941 : Egyptisch Leesboek, I. Teksten, Leyde.

-, 1948: Egyptian Readingbook, I. Exercises and Middle Egyptian Texts Selected and Edited, Leyde ( $3^{\mathrm{e}}$ éd. 1970).

Desroches Noblecourt Christiane, 1998 : «Le périple du "Naufragé” et le calendrier du Ramesseum », Memnonia, 9, p. 59-66.

DeSsOUDEIX Michel, 2016 : Lettres égyptiennes, III. La littérature du Moyen Empire, Arles.

Divin Marguerite, 1957 : Contes et légendes de l'Égypte ancienne, Paris.

DORN Andreas, 2013 : «Kulturelle Topografie literarischer Texte», dans G. MOERS et alii (éd.), Dating Egyptian Literary Texts, Hambourg, p. 73-109.

Dyroff Karl, PÖRTNER Balthasar, 1904 : Aegyptische Grabsteine und Denksteine aus süddeutschen Sammlungen, II, Munich.

EICHLER Eckhard, 2001 : «Zur Datierung und Interpretation der Lehre des Ptahhotep », Zeitschrift für Ägyptische Sprache und Altertumskunde, 128, p. 97-107. 
ENMARCH Roland, 2001 : "Of Spice and Mine: The Tale of the Shipwrecked Sailor and Middle Kingdom Expedition Inscriptions », dans F. HAGEN et alii (éd.), Narratives of Egypt and the Ancient Near East: Literary and Linguistic Approaches, (Orientalia Lovaniensia Analecta, 189), Leuven, p. 97-121.

ERMAN Adolf, 1906 : «Die Geschichte des Schiffbrüchigen», Zeitschrift für Ägyptische Sprache und Altertumskunde, 43, p. 1-26.

—, 1923 : Die Literatur der Ägypter, Leipzig.

—, 1927 : The Literature of the Ancient Egyptians (traduction de Aylward M. BLACKMAN), Londres.

FOSTER John L., 1984 : « Oriental Institute ostracon \#12074: "Menna's Lament” or "Letter to a Wayward Son" », Journal of the Society for the Study of Egypt Antiquities, 14, p. 8899.

—, 1988: " "The Shipwrecked Sailor": Prose or Verse? (Postponing Clauses and Tenseneutral Clauses) », Studien zur altägyptischen Kultur, 15, p. 69-109.

—, 1992 : Echoes of Egyptian Voices. An Anthology of Ancient Egyptian Poetry, Norman, Londres.

—, 2001 : Ancient Egyptian Literature. An Anthology, Austin.

Galán José Manuel, 1998 : Cuatro Viajes en la Literatura del Antiguo Egipto, Madrid.

—, 2005 : Four Journeys in Ancient Egyptian Literature (Lingua Aegyptia. Studia Monographica, 5), Göttingen.

GARDINER Alan Henderson, 1908 : «Inscriptions from the Tomb of Si-renpowet I., Prince of Elephantine », Zeitschrift für Ägyptische Sprache und Altertumskunde, 45, p. 123-140.

GARdiner Alan Henderson, PeET Thomas Eric, 1917: The Inscriptions of Sinai, I. Introduction and Plates (Egypt Exploration Fund, Memoir 36), Londres.

GARDINER Alan Henderson, PEET Thomas Eric, ČERNÝ Jaroslav, 1952 : The Inscriptions of Sinai, I. Introduction and Plates, $2^{\mathrm{e}}$ éd., Londres, Oxford.

-, 1955 : The Inscriptions of Sinai, II. Translations and Commentary (Egypt Exploration Society, Memoir 45), Londres, Oxford.

GoEDicke Hans, 1974 : Die Geschichte des Schiffbrüchigen (Ägyptologische Abhandlungen, 30), Wiesbaden.

GOLÉNISCHEFF Wladimir, 1882a: "Sur un ancien conte égyptien », Verhandlungen des fünften Internationalen Orientalisten-Congresses, 2.1, Berlin, p. 100-122.

- 1882b: «Un ancien conte égyptien (papyrus de Saint-Pétersbourg)», Revue égyptologique, 2, p. 341-344.

—, 1891 : Musée de l'Ermitage. Inventaire de la collection égyptienne, [Saint-Pétersbourg].

_, 1906 : «Le papyrus no. 1115 de l'Ermitage Impérial de Saint-Pétersbourg », Recueil de travaux relatifs à la philologie et à l'archéologie égyptiennes et assyriennes, 28, p. 73112.

—, 1912 : Le Conte du Naufragé (Bibliothèque d'étude IFAO, 2), Le Caire. 
—, 1913 : Les papyrus hiératiques $N^{\circ} N^{\circ} 1115,1116 \mathrm{~A}$ et $1116 \mathrm{~B}$ de l'Ermitage Impérial à $S^{T_{-}}$ Pétersbourg, Saint-Pétersbourg.

GRANDET Pierre, 1998 : Contes de l'Égypte ancienne, Paris.

GrIfFITH Francis Llewelyn, 1896 : dans C. D. WARNER, Specimen Pages of a Library of the World's Best Literature, XIII, New York, p. 5233-5236.

Guglielmi Waltraud, 1984 : «Zur Adaption und Funktion von Zitaten », Studien zur altägyptischen Kultur, 11, p. 347-364.

HAGEN Frederik, 2012 : An ancient Egyptian Literary Text in Context. The Instruction of Ptahhotep (Orientalia Lovaniensia Analecta, 218), Leuven.

HelcK Wolfgang, 1992 : «Die "Geschichte des Schiffbrüchigen”: eine Stimme der Opposition? », dans J. OsInG, E. K. NIELSEN (éd.), The Heritage of Ancient Egypt: Studies in Honour of Erik Iversen, Copenhague, p. 73-76.

Hilbig Anja, 2012 : « Die Geschichte des Schiffbrüchigen », dans C. Holler (éd.), Das Krokodil und der Pharao: eine Anthologie altägyptischer Literatur, Darmstadt, p. 39-45.

Hodel-Hoenes Sigrid, 1990 : «Betrachtungen zum Dank im Alten Ägypten », dans B. Schmitz, A. EgGeBrecht (éd.), Festschrift Jürgen von Beckerath: zum 70. Geburtstag am 19. Februar 1990 (Hildesheimer ägyptologische Beiträge, 30), Hildesheim, p. 119138.

Hodjash Svetlana, BeRlev Oleg, 1997 : « An Early Dynasty XII Offering Service from Meir (Moscow and London)", dans Essays in honour of Prof. Dr. Jadwiga Lipinska (Warsaw Egyptological Studies, 1), Varsovie, p. 283-290.

JANSSEN Jozef M. A., 1946 : De traditioneele egyptische autobiografie vóór het Nieuwe Rijk, Leyde.

JUNGE Friedrich, 1989: "Emphasis" and sentential meaning in Middle Egyptian (Göttinger Orientforschungen IV, 20), Wiesbaden.

KASTER Joseph, 1968 : Wings of the Falcon, New York.

KURTH Dieter, 1987 : «Zur Interpretation der Geschichte des Schiffbrüchigen », Studien zur altägyptischen Kultur, 14, p. 167-179.

LALOUETTE Claire, 1987 : Textes sacrés et textes profanes de l'ancienne Égypte: mythes, contes et poésie, II, Paris.

LAPIDUS Michel, 1995 : La quête de l'île merveilleuse (le conte du naufragé), Paris.

Le Guilloux Patrice, 1996 : Le Conte du Naufragé (Papyrus Ermitage 1115), Angers.

—, 2005 : Le Conte du Naufragé, $2^{\mathrm{e}}$ éd., Angers.

LEFEBVRE Gustave, 1949 : Romans et contes égyptiens de l'époque pharaonique, Paris.

LENZO-MARCHESE Giuseppina, 2004: "Les colophons dans la littérature égyptienne », Bulletin de l'Institut français d'archéologie orientale, 104, p. 359-376.

LichtheIM Miriam, 1973 : Ancient Egyptian Literature, I. The Old and Middle Kingdom, Berkeley, Los Angeles, Londres. 
LOPRIENO Antonio, 2003 : «Travel and Fiction in Egyptian Literature », dans D. O'ConNOR, S. Quirke (éd.), Mysterious Lands, Londres, p. 31-51.

MANUELIAN Peter Der, 1992 : «Interpreting "The Shipwrecked Sailor" », dans I. GAMERWALleRT, W. HelcK (éd.), Gegengabe: Festschrift für Emma Brunner-Traut, Tübingen, p. 223-233.

MASPERO Gaston, 1882 : Les Contes populaires de l'Égypte ancienne, Paris.

—, 1905 : Les Contes populaires de l'Égypte ancienne, $3^{\mathrm{e}}$ éd., Paris.

—, 1911 : Les Contes populaires de l'Égypte ancienne, $4^{\mathrm{e}}$ éd., Paris.

Mattha Girgis, 1959: "The Story of the Shipwrecked Sailor. Text and translation ", Cairo University. Bulletin of the Faculty of Arts, 21, p. 1-10. (non vidi)

MAthiEu Bernard, 2011 : «L'Enseignement de Ptahhotep », dans Visions d'Égypte. Émile Prisse d'Avennes (1807-1879), Paris, p. 62-85.

MODE Markus, 1980 : «Das Gleichnis des schiffbrüchigen Gefolgsmannes (Bemerkungen zu Papyrus 1115 der Staatlichen Ermitage in Leningrad) », Hallesche Beiträge zur Orientwissenschaft, 2, p. 5-57.

MoERs Gerald, 1999 : "Travel as narrative in Egyptian literature », dans G. MoERS (éd.), Definitely: Egyptian litérature: Proceedings of the symposion "Ancient Egyptian Literature: history and forms", Los Angeles, March 24-26, 1995 (Lingua aegyptia. Studia Monographica, 2), Göttingen, p. 43-61.

—, 2001 : Fingierte Welten in der ägyptischen Literatur des 2. Jahrtausends v. Chr.: Grenzüberschreitung, Reisemotiv und Fiktionalität (Probleme der Ägyptologie, 19), Leyde.

NAGAI Masakatsu, 2010: "On the Hieratic Script corresponding to the Hieroglyphic E9, E20/21, and E27: The case of Papyrus Ermitage No.1115 and Papyrus Prisse » (en japonais), Studies in Language and Literature: Language, 58, p. 43-54.

OBSOMER Claude, 1989: Les campagnes de Sésostris dans Hérodote (Connaissance de l’Égypte ancienne, 1), Bruxelles.

—, 1995: Sésostris Ir. Étude chronologique et historique du règne (Connaissance de l'Égypte ancienne, 5), Bruxelles.

—, 2007 : « Les expéditions d'Herkhouf (VI ${ }^{\mathrm{e}}$ dynastie) et la localisation de Iam », dans M.-C. Bruwier (éd.), Pharaons Noirs. Sur la Piste des Quarante Jours, Mariemont, p. 39-52.

—, 2017 : « Sésostris III et la frontière de Semna : analyse des stèles nubiennes de l'an 16 », BABELAO, 6, p. 1-38.

—, 2019a : «Les ports de la mer Rouge et l'expédition de Sésostris $\mathrm{I}^{\mathrm{er}}$ vers Pount », dans C. CANNUYER, La mer, les ports, les marins dans les civilisations orientales. Pauline Voûte et Robert Donceel in honorem (Acta Orientalia Belgica, 32), Bruxelles, p. 31-58.

—, 2019b : «Mersa Gaouasis sur la mer Rouge et les expéditions vers Pount au Moyen Empire », BABELAO, 8, p. 7-66.

PARKINSON Richard Bruce, 1991: "Teachings, Discourses and Tales from the Middle Kingdom », dans S. Quirke (éd.), Middle Kingdom Studies, New Malden, p. 91-122. 
—, 1997 : The Tale of Sinuhe and other Ancient Egyptian Poems: 1940-1640 BC, Oxford.

PETRIE W. M. Flinders, 1895, Egyptian Tales: translated from the papyri, I, Londres.

—, 1901 : dans E. WILSON (éd.), Egyptian Literature, New York, p. 173-179.

-, 1914 : dans E. M. Tappan (éd.), The World's Story: A History of the World in Story, Song and Art, III, Boston, p. 41-46.

PIEPER Max, 1927 : «Zur Datierung des Schiffbrüchigen », Orientalistische Literaturzeitung, 30, col. 737-738.

—, 1935 : Das ägyptische Märchen (Morgenland, 27), Leipzig.

PoE William Clay, 2008: The Writing of a Skillful Scribe. An introduction to hieratic Middle Egyptian through the text of The Shipwrecked Sailor, Santa Rosa.

POMEY Patrice, 2011 : «Les bateaux d'Ayn Soukhna. Les plus vieux vestiges de navires de mer actuellement connus », Égypte, Afrique \& Orient, 64, p. 3-12.

POSENER Georges, 1936 : La première domination perse en Égypte. Recueil d'inscriptions hiéroglyphiques (Bibliothèque d'étude IFAO, 11), Le Caire.

—, 1938 : «Le canal du Nil à la Mer Rouge avant les Ptolemées », Chronique d'Égypte, 13, p. $259-273$.

PoSTEL Lilian, 2008 : «Une nouvelle mention des campagnes nubiennes de Montouhotep II à Karnak », dans L. GABOLDE (éd.), Hommages à Jean-Claude Goyon (Bibliothèque d'étude IFAO, 143), Le Caire, p. 329-340.

QUIRKE Stephen, 2004 : Egyptian Literature 1800 BC (Golden House Publications. Egyptology, 2), Londres.

Ragazzoli Chloé, 2011 : «Fortunes du Papyrus Prisse», dans Visions d'Égypte. Émile Prisse d'Avennes (1807-1879), Paris, p. 86-91.

RedFord Donald B., 1973 : « The Tale of the Shipwrecked Sailor», dans A. K. GrAYson, D. B. REDFORD, Papyrus and Tablet, Englewood Cliffs, p. 54-58.

Rendsburg Gary A., 2000 : «Literary Devices in the Story of the Shipwrecked Sailor », Journal of the American Oriental Society, 120, p. 13-23.

RoBERSON Joshua, 2009 : " "Inside" or "at home"? An observation on Syntax in the Serpent's Prophesy, Shipwrecked Sailor, lines 167-169», Göttinger Miszellen, 222, p. 51-56.

ROEDER Günther, 1927 : Altägyptische Erzählungen und Märchen, Iena.

RoEDER Hubert, 2009 : «Die Erfahrung von Ba'u: "Sinuhe” und "Schiffbrüchiger" zwischen dem Erzählen und Lehren der 12. Dynastie », dans H. ROEDER (éd.), Das Erzählung in frühen Hochkulturen, I. Der Fall Ägypten, Munich, p. 75-157.

SAYED Abdel Monem A.H., 1983 : « New Light on the Recently Discovered Port on the Red Sea Shore », Chronique d'Égypte, 58, p. 23-37.

-, 2003 : "The Land of Punt: Problems of the Archaeology of the Red Sea and the Southeastern Delta », dans Z. HAwASS, L. PINCH BROCK (éd.), Egyptology at the dawn of the twenty-first century: proceedings of the Eighth International Congress of Egyptologists, Cairo, 2000, Le Caire, vol. I, p. 432-439. 
—, 2006 : « On the Non-Existence of the Nile-Red Sea Canal all over the Pharonic Time, and its Existence from the Persian Period Onwards », dans H. GYÖRY (éd.), Aegyptus et Pannonia II: Acta Symposii anno 2004, Budapest, p. 207-226.

Schотт Siegfried, 1950: Altägyptische Liebeslieder. Mit Märchen und Liebesgeschichten, $2^{\mathrm{e}}$ éd., Zürich.

SETHE Kurt, 1907 : «Bemerkungen zur "Geschichte des Schiffbrüchigen” », Zeitschrift für Ägyptische Sprache und Altertumskunde, 44, p. 80-87.

SEYFRIED Karl-Joachim, 1981 : Beiträge zu den Expeditionen des Mittleren Reiches in die Ost-Wüste (Hildesheimer ägyptologische Beiträge, 15), Hildesheim.

SIMPSON William Kelly, 1958: "Allusions to the Shipwrecked Sailor and the Eloquent Peasant in a Ramesside Text », Journal of the American Oriental Society, 78, p. 50-51.

—, 1972 : The Literature of Ancient Egypt, New Haven, Londres (2éd., 1973).

—, 1984 : « Schiffbrüchiger », dans Lexikon der Ägyptologie, V, col. 619-622.

—, 2003 : The Literature of Ancient Egypt, $3^{\mathrm{e}}$ éd., New Haven, Londres.

STASOW Wladimir, 1882 : « Jegipetskajaskarka otkrytaja w Petersburgskom Ermitaze » [Un Conte égyptien découvert à l'Ermitage de Saint-Pétersbourg], Vestnik Jewropy, 1, p. 580-602.

STAUDER Andréas, 2013 : Linguistic Dating of Middle Egyptian Texts (Lingua Aegyptia. Studia Monographica, 12), Hambourg.

STEINMANN Frank, 2008 : «Einige Bemerkungen zum Schiffbrüchigen », dans A. SPIEKERMANN (éd.), "Zur Zierde gereicht...": Festschrift Bettina Schmitz zum 60. Geburtstag am 24. Juli 2008 (Hildesheimer ägyptologische Beiträge, 50), Hildesheim, p. 251-256.

TALlet Pierre, 2012 : La zone minière pharaonique du Sud-Sinaï, 1. Catalogue complémentaire des inscriptions du Sinaï (Mémoires publiés par les membres de l'IFAO, 130), 2 vol., Le Caire.

_, 2018 : La zone minière pharaonique du Sud-Sinaï, 3. Les expéditions égyptiennes dans la zone minière du Sud-Sinaï du prédynastique à la fin de la XXe dynastie (Mémoires publiés par les membres de l'IFAO, 138), Le Caire.

TATERKA Filip, 2018 : Les expéditions au pays de Pount sous la XVIII dynastie égyptienne. Essai de compréhension du rôle idéologique des expéditions "commerciales », thèse de doctorat inédite, Poznan et Paris.

—, à paraître : «Hidden in Plain Sight, or Where to Look for the Mysterious Land of BiaPunt ».

VANDERSLEYEN Claude, 1990 : «En relisant le Naufragé », dans S. ISRAELIT-Groll, (éd.), Studies in Egyptology presented to Miriam Lichtheim, 2, Jérusalem, p. 1019-1024.

—, 1999 : Ouadj our, w’ $\underline{d}$ wr. Un autre aspect de la vallée du Nil (Connaissance de l'Égypte Ancienne, 7), Bruxelles.

VANDERSTEEN Laure-Anne, 2018 : Les inscriptions multilingues de Darius I $^{\text {er }}$ réalisées en Égypte, Louvain-la-Neuve, mémoire inédit de master. 
Vernus Pascal, 2001 : Sagesses de l'Égypte pharaonique, Paris.

—, 2013 : «La datation de L'Enseignement d'Aménemopé. Le littéraire et le linguistique », dans G. Moers et alii (éd.), Dating Egyptian Literary Text (Lingua Aegyptia, Studia Monographica, 11), Hambourg, p. 191-236.

VIKENTIEV Vladimir, 1935 : " The Metrical Scheme of the "Shipwrecked Sailor" », Bulletin de l'Institut français d'archéologie orientale, 35, p. 1-40.

VON BOMHARD Anne-Sophie, 1999: «Le conte du Naufragé et le papyrus Prisse », Revue d'Égyptologie, 50, p. 51-65.

WAINwRIGHT Gerald A., 1946 : «Zeberged: the Shipwrecked Sailor's Island », Journal of Egyptian Archaeology, 32, p. 31-38.

WeSTENDORF Wolfhart, 1990 : «Die Insel des Schiffbrüchigen - keine Halbinsel! », dans S. Israelit-Groll (éd.), Studies in Egyptology presented to Miriam Lichtheim, 2, Jérusalem, p. 1056-1064.

WiEDEMANN Alfred, 1906 : Altaegyptische Sagen und Märchen, Leipzig.

WIEDER Anja, 2007: Altägyptische Erzählungen. Form und Funktion einer literarischen Gattung, Berlin.

WILDUNG Dietrich, 1984 : L'âge d'or de l'Égypte. Le Moyen Empire, Fribourg.

WILKINSON Toby, 2016 : Writings from Ancient Egypt, Londres.

ŽÁBA Zbyněk, 1974: The Rock Inscriptions of Lower Nubia. Czechoslovak Concession, Prague. 


\title{
RÉSUMÉ
}

Le terme $\underline{h n w}$ est attesté à quatorze reprises dans le récit du Naufragé : 7 fois dans la préposition composée $m$ - $h n w, 7$ fois en tant que substantif. Rares sont les traducteurs de l'œuvre qui ont traduit de la même manière les 7 occurrences du nom $h n w$ : Résidence, pays, patrie, ... Cette hésitation sur le sens à lui conférer concerne surtout la première occurrence du terme, lorsque le bateau arrive à quai au terme de l'expédition de Nubie. Les marins sontils à Éléphantine, comme on l'a souvent cru, ou à la Résidence de Licht ? Cette étude permet de lever toute ambiguïté sur ce point important pour la compréhension de l'œuvre. C'est au port de Licht que le bateau se trouve amarré de retour de Nubie.

\begin{abstract}
The term $h n w$ is attested fourteen times in the story of the Shipwrecked: 7 times in the preposition composed $m$ - $h n w, 7$ times as a substantive. Few are the translators of the work who have translated in the same way the 7 occurrences of the name $h n w$ : Residence, home, country, ... This hesitation on the meaning to give it concerns especially the first occurrence of the term, when the boat arrives at quay at the end of the Nubian expedition. Are the sailors at Elephantine, as we often thought, or at the Residence in Licht? This study allows us to remove any ambiguity on this important point for understanding the work. It is at the port of Licht that the boat is docked back from Nubia.
\end{abstract}

